\title{
Models of q-algebra representations: Tensor products of special unitary and oscillator algebras
}

\author{
E. G. Kalnins \\ Department of Mathematics and Statistics, University of Waikato, Hamilton, New Zealand \\ H. L. Manocha \\ Indian Statistical Institute, New Delhi, India \\ Willard Miller, Jr. \\ School of Mathematics and Institute for Mathematics and its Applications, University of Minnesota, \\ Minneapolis, Minnesota 55455
}

(Received 2 August 1991; accepted for publication 10 February 1992)

\begin{abstract}
This paper begins a study of one- and two-variable function space models of irreducible representations of $q$ analogs of Lie enveloping algebras, motivated by recurrence relations satisfied by $q$-hypergeometric functions. The algebras considered are the quantum algebra $\mathrm{U}_{q}\left(\mathrm{su}_{2}\right)$ and a $q$ analog of the oscillator algebra (not a quantum algebra). In each case a simple one-variable model of the positive discrete series of finite- and infinitedimensional irreducible representations is used to compute the Clebsch-Gordan coefficients. It is shown that various $q$ analogs of the exponential function can be used to mimic the exponential mapping from a Lie algebra to its Lie group and the corresponding matrix elements of the "group operators" on these representation spaces are computed. It is shown that the matrix elements are polynomials satisfying orthogonality relations analogous to those holding for true irreducible group representations. It is also demonstrated that general $q$-hypergeometric functions can occur as basis functions in two-variable models, in contrast with the very restricted parameter values for the $q$-hypergeometric functions arising as matrix elements in the theory of quantum groups.
\end{abstract}

\section{INTRODUCTION}

In this paper we begin a study of function space models of irreducible representations of $q$ algebras. The algebras and models that we consider are motivated by recurrence relations satisfied by $q$-Jacobi, $q$-Laguerre, and $q$ Hermite polynomials. The point of view is that espoused in Ref. 1. Simple one-variable models of irreducible representations of the $q$ algebras are used to compute modelindependent properties of the representations, and these results are then applied to the more complicated twovariable models. In this approach $q$-hypergeometric functions depending on arbitrary complex parameters arise as basis functions in two-variable models. This contrasts with the results of the elegant theory of quantum groups, where special functions usually arise as matrix elements of quantum group operators. In the quantum group theory these spherical functions are very restricted classes of $q$-hypergeometric functions. ${ }^{2-6}$

In the Introduction we review the basic facts about the finite-dimensional irreducible representations of the quantum algebra $\mathrm{U}_{q}\left(\mathrm{su}_{2}\right)$, 4 , -10 and examine a model of these representations in which the representation space consists of polynomials in the complex variable $z .{ }^{11} \mathrm{We}$ use this model and a $q$ analog of the exponential function to give an alternate derivation of the Clebsch-Gordan coefficients for the tensor product of two irreducible representations. ${ }^{8,9,12}$

In Sec. II we review the basic facts about the positive discrete series of unitary irreducible representations of the quantum algebra analog of su( 1,1$).^{5,6,13}$ We study a onevariable model of these representations in which the Hilbert space consists of analytic functions on the unit disk. Again we use the model to give an alternate derivation of the Clebsch-Gordan decomposition.

In Sec. IV we introduce a $q$ analog of the fourdimensional oscillator Lie algebra. This $q$ analog is motivated by the recurrence relations for ${ }_{1} \phi_{1}$ basic hypergeometric functions, and is not a quantum algebra. Nevertheless, the model techniques still prove effective. We study a family of irreducible infinite-dimensional representations of this $q$ analog and find two distinct onevariable models: the first defined on a Hilbert space of functions analytic in the unit disk and the second ${ }^{11}$ on a Hilbert space of entire functions. In Sec. $V$ we use the disk model to work out the Clebsch-Gordan coefficients for the tensor product of two of these representations.

In Sec. VI we examine briefly the quantum algebra $W_{p}(1)$. We show that a particular representation of $W_{p}(1)$ can be identified with a particular representation of the algebra in Sec. IV, corresponding to a model of 
bosons, but, in general, these algebras are distinct. [On the other hand, if $\mathrm{U}_{q}\left(\mathrm{su}_{2}\right)$ and $W_{p}(1)$ are considered as complex algebras with identities, then $\mathrm{U}_{p^{1 / 2}}\left(\mathrm{su}_{2}\right) \equiv W_{p}(1)$.]

In Sec. VII we give some examples showing how the various models of $q$-algebra representations can be used to derive identities obeyed by $q$ series associated with the models. We draw our examples from the oscillator algebra of Sec. IV, though the ideas apply generally. Using two more $q$ analogs of the exponential function we mimic the exponential mapping from a Lie algebra to its Lie group and compute the matrix elements of "group operators" with respect to a standard basis in the representation space. Depending on which $q$ analog of the exponential we employ we obtain various $q$ analogs of the associated Laguerre polynomials $L_{n}^{(m-n)}(x)$ for $m, n$ non-negative integers. We demonstrate that these matrix elements themselves form bases for two-variable models of irreducible representations of the oscillator algebra and that indeed they are special cases of models involving $q$ analogs of the Laguerre polynomials $L_{n}^{(\gamma-n)}(x)$ for general real $\gamma$. We show that the polynomials in each example satisfy orthogonality relations that are $q$ analogs of the orthogonality relations for matrix elements of irreducible representations of the oscillator group.

In forthcoming papers we will extend the ideas in Sec. VII, explore the discrete orthogonality and biorthogonality relations for $q$ analogs of Laguerre and other polynomials, obtained by multiplying the matrix of a "group operator" by its inverse matrix, and explore the various identities that arise from a knowledge of these matrices and the Clebsch-Gordan coefficients.

For the most part, the notation used for the $q$ series in this paper follows that of Gasper and Rahman. ${ }^{14}$

\section{MODELS OF FINITE-DIMENSIONAL $U_{q}\left(\mathrm{su}_{2}\right)$ REPRESENTATIONS}

The quantum algebra $\mathrm{U}_{q}\left(\mathrm{su}_{2}\right)$ is the associative algebra generated by the elements $H, E_{+}, E_{-}$, which obey the commutation relations

$$
\begin{aligned}
& {\left[H, E_{+}\right]=E_{+}, \quad\left[H, E_{-}\right]=-E_{-},} \\
& {\left[E_{+}, E_{-}\right]=\frac{q^{H}-q^{-H}}{q^{1 / 2}-q^{-1 / 2}} .}
\end{aligned}
$$

Here we take $q$ to be a real parameter, such that $0<q<1$. In the limit as $q \rightarrow 1$ relations (2.1) go to the usual commutation relations for the complexification of the Lie algebra $\mathrm{su}_{2}$. Finite-dimensional irreducible representations of $\mathrm{U}_{q}\left(\mathrm{su}_{2}\right)$ are determined by the integral or half-integral number $u: 2 u=0,1,2, \ldots$. The corresponding representation $D(2 u)$ is defined on the $(2 u+1)$-dimensional Hilbert space $H_{2 u}$ with orthonormal basis $\left\{e_{m}: m=-u\right.$, $-u+1, \ldots, u\}$, such that

$$
\begin{aligned}
& E_{+} e_{m}=\left([u-m]_{q}[u+m+1]_{q}\right)^{1 / 2} e_{m+1}, \\
& E_{-} e_{m}=\left([u+m]_{q}[u-m+1]_{q}\right)^{1 / 2} e_{m-1}, \\
& H e_{m}=m e_{m},
\end{aligned}
$$

where

$$
[m]_{q}=\frac{q^{m / 2}-q^{-m / 2}}{q^{1 / 2}-q^{-1 / 2}}=q^{-(m-1) / 2}\left(\frac{1-q^{m}}{1-q}\right) .
$$

A second convenient basis for $H_{2 u}$ is the set $\left\{f_{n}: n\right.$ $=0,1, \ldots, 2 u\}$, such that

$$
\begin{aligned}
& E_{+} f_{n}=-q^{-1}[2 u-n]_{q} f_{n+1}, \\
& e_{-} f_{n}=-q[n]_{q} f_{n-1}, \\
& H f_{n}=(-u+n) f_{n} .
\end{aligned}
$$

Here

$$
f_{n}=\left[\frac{(-1)^{n} q^{(3 / 2-u) n}(q ; q)_{n}}{\left(q^{-2 u} ; q\right)_{n}}\right]^{1 / 2} e_{-u+n}
$$

Since the element

$$
C=E_{+} E_{-}+\frac{q^{H-1 / 2}+q^{-H+1 / 2}}{\left(q^{1 / 2}-q^{-1 / 2}\right)^{2}}
$$

commutes with each generator of $U_{q}\left(s_{2}\right)$, it corresponds to a multiple of the identity operator $I$ on $H_{2 u}$. Indeed,

$$
C=\frac{q^{u+1 / 2}+q^{-u-1 / 2}}{\left(q^{1 / 2}-q^{-1 / 2}\right)^{2}} I
$$

on $H_{2 u}$.

Given the irreducible representations $D\left(2 u_{1}\right)$ and $D\left(2 u_{2}\right)$ on the spaces $H_{2 u_{1}}$ and $H_{2 u_{2}}$, respectively, we define the tensor product representation $D\left(2 u_{1}\right) \otimes_{a} D\left(2 u_{2}\right)$ of $\mathrm{U}_{q}\left(\mathrm{su}_{2}\right)$ on the space $H_{2 u_{1}} \otimes H_{2 u_{2}}$ by the operators

$$
\begin{aligned}
& F_{+}=\Delta_{a}\left(E_{+}\right)=E_{+} \otimes q^{a H}+q^{(a-1) H} \otimes E_{+}, \\
& F_{-}=\Delta_{a}\left(E_{-}\right)=E_{-} \otimes q^{(1-a) H}+q^{-a H} \otimes E_{-}, \\
& L=\Delta_{a}(H)=H \otimes I+I \otimes H,
\end{aligned}
$$

where $a$ is a real number. The operators $F_{ \pm}, L$ satisfy the same commutation relations as the operators $E_{ \pm}, H$ :

$$
\left[L, F_{ \pm}\right]= \pm F_{ \pm}, \quad\left[F_{+}, F_{-}\right]=\frac{q^{L}-q^{-L}}{q^{1 / 2}-q^{-1 / 2}}
$$


(If we require that $F_{+}$is the adjoint of $F_{-}$and $L$ is self-adjoint, then we must have $a=\frac{1}{2}$, which is the usual definition of the tensor product; see Refs. 7 and 8. Thus the interest of representations with general $a$ relates primarily to nonunitary representations, particularly the nonunitary infinite-dimensional representations to be considered in the next section. However, we will work out the details of the Clebsch-Gordan decomposition of $a$ also in the finite-dimensional case.) It is straightforward to show that

$$
q^{-\alpha H \otimes H} \Delta_{\alpha} q^{\alpha H \otimes H}=\Delta_{a-\alpha}
$$

so

$$
q^{(b-1 / 2) H \otimes H} \Delta_{1 / 2} q^{(1 / 2-b) H \otimes H}=\Delta_{b}
$$

and the operators $\Delta_{b}$ are equivalent to the operators $\Delta_{1 / 2}$.

In order to decompose $D\left(2 u_{1}\right) \otimes_{a} D\left(2 u_{2}\right)$ into irreducible subspaces, we introduce a convenient onevariable model of $D(2 u) .{ }^{11}$ Here the vector space $H_{2 u}$ consists of polynomials $f(z)$ of maximum order $2 u$ in the complex variable $z$. The action of $\mathrm{U}_{q}\left(\mathrm{su}_{2}\right)$ is defined by the operators

$$
\begin{aligned}
& E_{+}=\frac{q^{-1 / 2} z}{1-q}\left(q^{u} T_{z}^{-1 / 2}-q^{-u} T_{z}^{1 / 2}\right), \\
& E_{-}=\frac{q^{3 / 2}}{z(1-q)}\left(T_{z}^{1 / 2}-T_{z}^{-1 / 2}\right), \\
& H=-u+z \frac{d}{d z}
\end{aligned}
$$

where $T_{z} f(z)=f(q z)$. The basis functions $\left\{f_{n}=z^{n}: n\right.$ $=0,1, \ldots, 2 u\}$ satisfy relations $(2.4)$. We define a bilinear form $\langle\cdot, \cdot\rangle$ on $H_{2 u}$, such that

$$
\langle f, g\rangle=\iint_{-\infty}^{\infty} f(z) g(\bar{z}) \rho(z, \bar{z}) d x d y,
$$

where $z=x+i y$ and $d x d y=-(i / 2) d z d \bar{z}$. Further, we require that

$$
\left\langle E_{+} f, g\right\rangle=\left\langle f, E_{-} g\right\rangle, \quad\langle H f, g\rangle=\langle f, H g\rangle,
$$

for all $f, g \in H_{2 u}$. A straightforward computation gives $\rho(z, \bar{z}) \equiv \rho(z \bar{z})=\rho(w)$, where

$$
\rho(q w)=\frac{\left(1+q^{-u-5 / 2} w\right)}{\left(1+q^{u-1 / 2} w\right)} \rho(w)
$$

or

$$
\rho(w)=K \frac{\left(-w q^{u-1 / 2} ; q\right)_{\infty}}{\left(-w q^{-u-5 / 2} ; q\right)_{\infty}}=\frac{K}{\left(-w q^{-u-5 / 2} ; q\right)_{2 u+2}} .
$$

Since

$$
\int_{0}^{\infty} \frac{d w}{\left(-w q^{-u-5 / 2} ; q\right)_{2 u+2}}=\frac{q^{u+5 / 2} \ln q^{-1}}{\left(1-q^{2 u+1}\right)},
$$

we choose

$$
K=\frac{1-q^{2 u+1}}{q^{u+5 / 2} \ln q^{-1}},
$$

so that $\langle 1,1\rangle=\left\langle f_{0}, f_{0}\right\rangle=1$. The functions

$$
\begin{aligned}
e_{m}(z) & =\frac{(q ; q)_{2 u}^{1 / 2} z^{u+m}}{\left(q^{(u+3 / 2)(u+m)} q^{-(u+m)(u+m-4) / 2}(q ; q)_{u+m}(q ; q)_{u-m}\right)^{1 / 2}} \\
& =z^{u+m} \sqrt{\frac{\left(q^{-2 u} ; q\right)_{u+m}}{\left(-q^{3 / 2-u}\right)^{u+m}(q ; q)_{u+m}}}, \quad m=-u,-u+1, \ldots, u,
\end{aligned}
$$

form an orthonormal basis for $H_{2 u}$ in this model. This Hilbert space has the kernel function

$$
S\left(\bar{z}^{\prime}, z\right)=\sum_{m=-u}^{u} e_{m}\left(\bar{z}^{\prime}\right) e_{m}(z)=\left(-q^{-u-3 / 2} \bar{z}^{\prime} z ; q\right)_{2 u},
$$

so that

$$
\left\langle g, S\left(\bar{z}^{\prime}, \cdot\right)\right\rangle=g\left(z^{\prime}\right)
$$

for $z^{\prime} \in \mathbb{C}$ and $g \in H_{2 u}$.
It follows from (2.8) and (2.10) that the operators correspond to the tensor product $D\left(2 u_{1}\right) \otimes_{a} D\left(2 u_{2}\right)$ take the form

$$
\begin{aligned}
F_{+}= & \frac{1}{1-q}\left\{q^{-a u_{2}-1 / 2} z\left(q^{u_{1}} T_{z}^{-1 / 2}-q^{-u_{1}} T_{z}^{1 / 2}\right) T_{w}^{a}\right. \\
& \left.+q^{(-a+1) u_{1}-1 / 2} w\left(q^{u_{2}} T_{w}^{-1 / 2}-q^{-u_{2}} T_{w}^{1 / 2}\right) T_{z}^{-1+a}\right\},
\end{aligned}
$$




$$
\begin{aligned}
& F_{-}= \frac{q^{3 / 2}}{1-q}\left\{\frac{q^{(-1+a) u_{2}}}{z}\left(T_{z}^{1 / 2}-T_{z}^{-1 / 2}\right) T_{w}^{-a+1}\right. \\
&\left.+\frac{q^{a u_{1}}}{w}\left(T_{w}^{1 / 2}-T_{w}^{-1 / 2}\right) T_{z}^{-a}\right\} \\
& L=-u_{1}-u_{2}+z \frac{d}{d z}+w \frac{d}{d w} .
\end{aligned}
$$

The functions

$$
p_{k, l}(z, w)=z^{k} w^{l}, \quad k=0,1, \ldots, 2 u_{1} ; \quad l=0,1, \ldots, 2 u_{2}
$$

form a basis for $H_{2 u_{1}} \otimes H_{2 u_{2}}$. We use the method of highest weights to decompose $H_{2 u_{1}} \otimes H_{2 u_{2}}$ into irreducible subspaces. The eigenvectors of $L$ such that $F, f=0$ are given by

$$
\begin{aligned}
f_{s, 0}^{a}= & \sum_{r=0}^{s} \frac{\left(q^{-s} ; q\right)_{r}}{(q ; q)_{r}} q^{-(-2 a-1) r^{2} / 2} z^{s} \\
& \times\left[\frac{w}{z} q^{-(-1-a)\left(u_{2}-a u_{1}+(a+1 / 2) s\right.}\right]^{r}
\end{aligned}
$$

Note that in the case $a=\frac{1}{2}$, expression (2.16) can be summed explicitly:

$$
\begin{aligned}
f_{s, 0} & =z^{s} \frac{\left((w / z) q^{-(1 / 2) u_{1}-(1 / 2) u_{2}} ; q\right)_{\infty}}{\left(w / z q^{-1 / 2 u_{1}-1 / 2 u_{2}+s} ; q\right)_{\infty}} \\
& =z^{s}\left(\frac{w}{z} q^{-(1 / 2) u_{1}-(1 / 2) u_{2}} ; q\right), \\
L f_{s, 0} & =\left(s-u_{1}-u_{2}\right) f_{s, 0}, \quad s=0,1, \ldots, \min \left(2 u_{1}, 2 u_{2}\right) .
\end{aligned}
$$

We introduce a bilinear form $\langle\cdot, \cdot\rangle_{a}$ on $H_{2 u_{1}}$ $\otimes H_{2 u_{2}}$, such that

$$
\begin{aligned}
\left\langle p_{\left.k_{1}, l_{1}, p_{k_{2}, l_{2}}\right\rangle_{a}}\right. & \\
= & \delta_{k_{1} k_{2}} \delta_{l_{1} l_{2}}(-1)^{k_{1}+l_{1}} q^{(3 / 2)\left(k_{1}+l_{1}\right)-u_{1} k_{1}-u_{2} l_{1}} \\
& \times q^{(2 a-1)\left(u_{1} l_{1}+u_{2} k_{1}-k_{1} l_{1}\right)} \frac{(q ; q)_{k_{1}}(q ; q)_{l_{1}}}{\left(q^{-2 u_{1}} ; q\right)_{k_{1}}\left(q^{-2 u_{2}} ; q\right)_{l_{1}}} .
\end{aligned}
$$

By construction,

$$
\left\langle F_{+} p_{1}, p_{2}\right\rangle_{a}=\left\langle p_{1}, F_{-} p_{2}\right\rangle_{a}, \quad\left\langle L p_{1}, p_{2}\right\rangle_{a}=\left\langle p_{1}, L p_{2}\right\rangle_{a},
$$

for all $p_{1}, p_{2} \in H_{2 u_{1}} \otimes H_{2 u_{2}}$. [For $a=\frac{1}{2}$, this agrees with the inner product on $H_{2 u_{1}} \otimes H_{2 u_{2}}$ induced by (2.12) and (2.13).] A straightforward computation yields

$$
\begin{aligned}
\left\langle f_{s, 0}^{a} f_{s, 0}^{a}\right\rangle_{a}= & (-1)^{s} q^{-u_{1} s+(2 a-1) u_{2} s+(3 / 2) s} \\
& \times \frac{(q ; q)_{s}\left(q^{-2 u_{2}-2 u_{1}+s-1} ; q\right)_{s}}{\left(q^{-2 u_{1}} ; q\right)_{s}\left(q^{-2 u_{2}} ; q\right)_{s}} .
\end{aligned}
$$

Now we define vectors $f_{s, k}$, recursively, by

$$
\begin{gathered}
f_{s, k+1}=\frac{-q}{\left[2 u_{1}+2 u_{2}-2 s-k\right]_{q}} F_{+} f_{s, k} \\
k=0,1, \ldots, 2 u_{1}+2 u_{2}-2 s-1, \\
s=0,1, \ldots, \min \left(2 u_{1}, 2 u_{2}\right) .
\end{gathered}
$$

Using the recurrence relations (2.9) and the Casimir operator

$$
C^{\prime}=F_{+} F_{-}+\frac{q^{L-1 / 2}+q^{-L+1 / 2}}{\left(q^{1 / 2}-q^{-1 / 2}\right)^{2}}
$$

we can verify the following.

Lemma 1:

$$
\begin{aligned}
& \text { (1) } F_{+} f_{s, k}=-q^{-1}\left[2 u_{1}+2 u_{2}-2 s-k\right]_{q} f_{s, k+1} \text {, } \\
& \text { (2) } F f_{s, k}=-q[k]_{q} f_{s, k-1}, \\
& \text { (3) } L f_{s, k}=\left(-u_{1}-u_{2}+s+k\right) f_{s, k}
\end{aligned}
$$

In particular, $F_{+} f_{s, 2 u_{1}+2 u_{2}-2 s}=0$. For fixed $s$ the $\left\{f_{s, k}\right\}$ form an orthogonal basis for a subspace of $H_{2 u_{1}} \otimes H_{2 u_{2}}$ transforming according to the irreducible representation $D\left(2 u_{1}+2 u_{2}-2 s\right)$.

\section{Lemma 2:}

$$
D\left(2 u_{1}\right) \otimes D\left(2 u_{2}\right) \cong \sum_{s=0}^{\min \left(2 u_{1}, 2 u_{2}\right)} \oplus D\left(2 u_{1}+2 u_{2}-2 s\right)
$$

Lemma 3:

$$
\begin{aligned}
& \left\langle f_{s, k}^{a} f_{s^{\prime}, k^{\prime}}^{a}\right\rangle \\
& =\delta_{s s^{\prime}} \delta_{k k^{\prime}}
\end{aligned}
$$

$$
\times(-1)^{k+s} q^{-u_{1} s+(2 a-1) u_{2} s+(3 / 2) s+k\left(-u_{1}-u_{2}+s+3 / 2\right)}
$$

$$
\times \frac{(q ; q)_{k}(q ; q)_{s}\left(q^{-2 u_{1}-2 u_{2}+s-1} ; q\right)_{s}}{\left(q^{-2 u_{1}} ; q\right)_{s}\left(q^{-2 u_{2}} ; q\right)_{s}\left(q^{-2 u_{1}-2 u_{2}+2 s} ; q\right)_{k}}
$$


Instead of the orthogonal basis $\left\{f_{s, k}\right\}$ we can pass to the orthonormal basis $\left\{e_{m}^{v}\right\}$, where

$$
e_{m}^{v}=\left\|f_{s, k}\right\|^{-1} f_{s, k}
$$

and $v=u_{1}+u_{2}-s, m=-u_{1}-u_{2}+s+k$, so that

$$
\begin{aligned}
& v=u_{1}+u_{2}, u_{1}+u_{2}-1, \ldots,\left|u_{1}-u_{2}\right|, \\
& m=-v,-v+1, \ldots, v .
\end{aligned}
$$

To derive a generating function for the ClebschGordan coefficients we apply a $q$ analog of the exponential of $F_{+}$to $f_{s, 0}^{a}$ and expand the resulting expression in terms of the monomial $p_{n, m}$ basis:

$$
\left(\exp _{q} t F_{+}\right) f_{s, 0}^{a}=\sum_{k=0}^{\infty} \frac{q^{k(k+1) / 4}}{(q ; q)_{k}} t^{k} F_{+}^{k} f_{s, 0}^{a} .
$$

To compute the right-hand side of $(2.23)$ we need to evaluate the terms $F_{+}^{k} p_{n, m}$, where $p_{n, m}=z^{n} w^{m}$ and

$$
F_{+}=Y+X, \quad Y=E_{+} \otimes q^{a H}, \quad X=q^{(a-1) H} \otimes E_{+} .
$$

Note that $Y X=q X Y$. Moreover, a straightforward induction argument using this property (see Refs. 4 and 14, p. 28], yields the following.

\section{Lemma 4:}

$$
(Y+X)^{k}=\sum_{l=0}^{k} \frac{(q ; q)_{k}}{(q ; q)_{l}(q ; q)_{k-l}} X^{l} Y^{k-l}
$$

The right-hand side of this expression is easily evaluated on the $p_{n, m}$ basis. Then, making use of (2.16), we obtain the expression

$$
\begin{aligned}
\left(\exp _{q} t F_{+}\right) f_{s, 0}^{a}= & \sum_{h=0}^{s} \sum_{j, l=0}^{\infty} \frac{\left(q^{-s} ; q\right)_{h}\left(q^{-2 u_{1}+s-h} ; q\right)_{j}\left(q^{-2 u_{2}+h} ; q\right)_{l}}{(q ; q)_{h}(q ; q)_{j}(q ; q)_{l}(1-q)^{j+l}} q^{(a-1 / 2)\left(l j-l h-h^{2}\right)+(a+1 / 2) h j} \\
& \times q^{l\left([1 \cdots a]\left[u_{1}-s\right]+u_{2}\right)+j\left(u_{1}-a u_{2}-s / 2\right)+h\left([a-1] u_{2}-a u_{1}+[a+1 / 2] s\right)^{s-h+j}} z^{h+l} t^{j+l}
\end{aligned}
$$

where $k=j+l$. On the other hand, from (2.21),

$$
\left(\exp _{q} t F_{+}\right) f_{s, 0}^{a}=\sum_{k=0}^{\infty} \frac{q^{k(k+1) / 4}}{(q ; q)_{k}} t^{k} F_{+}^{k} f_{s, 0}^{a}=\sum_{k=0}^{\infty} \frac{\left(q^{-2 u_{1}-2 u_{2}+2 s} ; q\right)_{k}}{(q ; q)_{k}}\left(\frac{t q^{u_{1}+u_{2}-s}}{1-q}\right)^{k} f_{s, k}^{a}
$$

Thus

$$
\begin{aligned}
f_{s, k}^{a}(z, w)= & \sum_{r=0}^{s+k} \frac{q^{k\left[-(a+1) u_{2}+s / 2\right]}(q ; q)_{k}\left(q^{-2 u_{1}} ; q\right)_{s+k-r}\left(q^{-2 u_{2}} ; q\right)_{r}}{(q ; q)_{r}(q ; q)_{\infty}\left(q^{2 s-2 u_{1}-2 u_{2}} ; q\right)_{k}\left(q^{-2 u_{1}} ; q\right)_{s}} \\
& \times\left(q^{1+k-r} ; q\right)_{\infty} q^{r\left[(a-1 / 2)(k-r)+(a-1 / 2) s-a\left(u_{1}-u_{2}\right)+u_{2}\right]} \\
& \times{ }_{3} \phi_{2}\left(\begin{array}{c}
q^{-s}, q^{2 u_{1}-s+1}, q^{-r} \\
q^{k-r+1}, q^{-2 u_{2}} ; q ; q^{2 s-2 u_{1}-2 u_{2}+k}
\end{array}\right) z^{s+k-r} w^{r}
\end{aligned}
$$

From this result we can easily expand the orthonormal basis $\left\{e_{m}^{v}\right\}$ for $H_{2 u_{1}} \otimes H_{2 u_{2}}$,

$$
\begin{aligned}
f_{s, k}^{a}= & {\left[\frac{(-1)^{u_{1}+u_{2}-v}(q ; q)_{u_{1}+u_{2}-v}\left(q^{-u_{1}-u_{2}-v-1} ; q\right)_{u_{1}+u_{2}-v}(q ; q)_{v+m}(q ; q)_{v-m}}{\left(q^{-2 u_{1}} ; q\right)_{u_{1}+u_{2}-v}\left(q^{-2 u_{2}} ; q\right)_{u_{1}+u_{2}-v}(q ; q)_{2 v}}\right]^{1 / 2} } \\
& \times q^{1 / 4\left(v^{2}-m^{2}\right)+(m+v)-(1 / 2) u_{1}\left(u_{1}+u_{2}-v\right)+(3 / 4)\left(u_{1}+u_{2}-v\right)+(a-1 / 2) u_{2}\left(u_{1}+u_{2}-v\right)} e_{m}^{v},
\end{aligned}
$$

where

$$
m=v, v-1, \ldots,-v ; \quad v=u_{1}+u_{2}, u_{1}+u_{2}-1, \ldots,\left|u_{1}-u_{2}\right|,
$$

in terms of the orthonormal basis (2.18), 


$$
\begin{aligned}
e_{n_{1}}^{u_{1}} \otimes a_{a} e_{n_{2}}^{u_{2}}= & {\left[\frac{\left(q^{-2 u_{1}} ; q\right)_{u_{1}+n_{1}}\left(q^{-2 u_{2}} ; q\right)_{u_{2}+n_{2}}}{(q ; q)_{u_{1}+n_{1}}(q ; q)_{u_{2}+n_{2}}(-1)^{u_{1}+u_{2}+n_{1}+n_{2}}}\right]^{1 / 2} q^{(1 / 2-a)\left[u_{1}\left(u_{1}+n_{1}\right)+u_{2}\left(u_{2}+n_{2}\right)-\left(u_{1}+n_{1}\right)\left(u_{2}+n_{2}\right)\right]} } \\
& \times q^{-(3 / 4)\left(u_{1}+u_{2}+n_{1}+n_{2}\right)+\left(u_{1} / 2\right)\left(u_{1}+n_{1}\right)+\left(u_{2} / 2\right)\left(u_{2}+n_{2}\right)} p_{u_{1}+n_{1}, u_{2}+n_{2} ;} n_{i}=u_{i}, u_{i}-1, \ldots,-u_{i}: \\
e_{m}^{v}= & \sum_{n_{1}, n_{2}} a\left[\begin{array}{lll}
u_{1} & u_{2} & v \\
n_{1} & n_{2} & m
\end{array}\right]_{q}^{e_{n_{1}}^{u_{1}} \otimes{ }_{a} e_{n_{2}}^{u_{2}}}
\end{aligned}
$$

This defines the Clebsch-Gordan coefficients for the tensor product $D\left(2 u_{1}\right) \otimes D\left(2 u_{2}\right)$. It follows from (2.27) that these coefficients vanish unless $m=n_{1}+n_{2}$. Furthermore, the orthogonality of the two bases implies the identities

$$
\begin{gathered}
\sum_{n_{1}, n_{2}}\left[\begin{array}{lll}
u_{1} & u_{2} & v \\
n_{1} & n_{2} & m
\end{array}\right]_{q a}\left[\begin{array}{lll}
u_{1} & u_{2} & v^{\prime} \\
n_{1} & n_{2} & m
\end{array}\right]_{q}^{*}=\delta_{v v^{\prime}} \\
\sum_{v a}\left[\begin{array}{lll}
u_{1} & u_{2} & v \\
n_{1} & n_{2} & m
\end{array}\right]_{q a}\left[\begin{array}{lll}
u_{1} & u_{2} & v^{\prime} \\
n_{1}^{\prime} & n_{2}^{\prime} & m
\end{array}\right]_{q}^{*}=\delta_{n_{1} n_{1}^{\prime}}
\end{gathered}
$$

(In the second sum we require $n_{1}+n_{2}=n_{1}^{\prime}+n_{2}^{\prime}=m$, and * is complex conjugation.) From (2.27) $-(2.30)$ we find

$$
\begin{aligned}
& { }_{a}\left[\begin{array}{ccc}
u_{1} & u_{2} & v \\
n_{1} & n_{2} & m
\end{array}\right]_{q}=\left[\frac{\left(q^{-2 u_{1}} ; q\right)_{u_{1}+u_{2}-v}\left(q^{-2 u_{2}} ; q\right)_{u_{1}+u_{2}-v}\left(q^{-2 u_{1}} ; q\right)_{u_{1}+n_{1}}\left(q^{-2 u_{2}} ; q\right)_{u_{2}+n_{2}}(q ; q)_{v+m}(q ; q)_{u_{1}+n_{1}}}{(q ; q)_{u_{1}+u_{2}-v}\left(q^{-u_{1}-u_{2}-v-1} ; q\right)_{u_{1}+u_{2}-v}\left(q^{-2 v} ; q\right)_{v+m}(q ; q)_{u_{2}+n_{2}}}\right]^{1 / 2} \\
& \times \frac{\left(q^{n_{1}+v-u_{2}+1} ; q\right)_{\infty}}{(q ; q)_{\infty}\left(q^{-2 u_{1}} ; q\right)_{u_{1}+u_{2}-v}} q^{\alpha\left(u_{1}-u_{2}\right)\left(u_{1}+u_{2}+n_{1}-n_{2}\right.} \\
& \times q^{1 / 2\left[-u_{1}^{2}+u_{2}^{2}+2 u_{1} u_{2}-2 u_{2} v-n_{1} u_{1}+n_{2} u_{1}-n_{1} u_{2}-n_{2} u_{2}\right]} \\
& \times_{3} \phi_{2}\left(\begin{array}{ccc}
q^{v-u_{1}-u_{2}}, & q^{u_{1}-u_{2}+v+1}, & q^{-u_{2}-n_{2}} \\
q^{v+n_{1}-u_{2}+1}, & q^{-2 u_{2}} & ; q ; q^{-v+m}
\end{array}\right)
\end{aligned}
$$
236):

In the case $a=\frac{1}{2}$ the sum (2.25) can be evaluated explicitly (through use of the $q$-Vandermonde identity; Ref. 14, p.

$$
\left(\exp _{q} t F_{+}\right) f_{s, 0}^{1 / 2}=\frac{\left([z t /(1-q)] q^{-u_{2} / 2+s / 2} ; q\right)_{\infty}\left([w t /(1-q)] q^{u_{1} / 2-u_{2}+s / 2} ; q\right)_{\infty}\left((w / z) q^{-u_{1} / 2-u_{2} / 2} ; q\right)_{s}}{\left([w t /(1-q)] q^{u_{1} / 2+u_{2}-s / 2} ; q\right)_{\infty}\left([z t /(1-q)] q^{u_{1}-u_{2} / 2-s / 2} ; q\right)_{\infty}}
$$

Thus, from (2.26), (2.28), and (2.29) we obtain the generating function (after some rescaling)

$$
\begin{aligned}
x_{2}^{u_{3}+u_{2}-u_{1}}\left(\frac{x_{3}}{x_{2}} q^{u_{1}-u_{2}-u_{3}} ; q\right)_{u_{2}+u_{3}-u_{1}} x_{3}^{u_{3}+u_{1}-u_{2}}\left(\frac{x_{1}}{x_{3}} q^{u_{2}-u_{3}-u_{1}} ; q\right)_{u_{3}+u_{1}-u_{2}} x_{1}^{u_{1}+u_{2}-u_{3}}\left(\frac{x_{2}}{x_{1}} q^{1+u_{3}-u_{1}-u_{2}} ; q\right)_{u_{1}+u_{2}-u_{3}} \\
=(-1)^{u_{3}+u_{2}-u_{1}} q^{(1 / 2)\left(u_{1}+u_{2}-u_{3}\right)+u_{3}\left(u_{1}+u_{2}\right)-(1 / 2)\left(u_{1}^{2}+u_{2}^{2}+u_{3}^{2}\right)} \\
\quad \times\left[\frac{(-1)^{u_{1}+u_{2}-u_{3}}(q ; q)_{u_{1}+u_{2}-u_{3}}\left(q^{-u_{1}-u_{2}-u_{3}-1} ; q\right)_{u_{1}+u_{2}-u_{3}}}{\left(q^{-2 u_{1}} ; q\right)_{u_{1}+u_{2}-u_{3}}\left(q^{-2 u_{2}} ; q\right)_{u_{1}+u_{2}-u_{3}}}\right]^{1 / 2}
\end{aligned}
$$




$$
\begin{aligned}
& \times \sum_{n_{1}, n_{2}}(-1)^{u_{3}+m} x_{3}^{u_{3}-m} x_{1}^{u_{1}+n_{1}} x_{2}^{u_{2}+n_{2}} q^{1 / 2\left[m\left(u_{2}-u_{1}+u_{3}\right)+n_{1}\left(u_{2}-u_{3}+u_{1}\right)+n_{2}\left(u_{3}-u_{1}+u_{2}+2\right)\right]} \\
& \times\left[\frac{\left(q^{-2 u_{3}} ; q\right)_{u_{3}+m}\left(q^{-2 u_{1}} ; q\right)_{u_{1}+n_{1}}\left(q^{-2 u_{2}} ; q\right)_{u_{2}+n_{2}}(-1)^{u_{3}-u_{1}-u_{2}}}{(q ; q)_{u_{3}+m}(q ; q)_{u_{1}+n_{1}}(q ; q)_{u_{2}+n_{2}}}\right]^{1 / 2}\left[\begin{array}{lll}
u_{1} & u_{2} & u_{3} \\
n_{1} & n_{2} & m
\end{array}\right]_{q},
\end{aligned}
$$

where $m=n_{1}+n_{2}$. The left-hand side of (2.34) admits symmetries, which account for the 72 symmetries of the Clebsch-Gordan coefficients. ${ }^{8}$ Indeed, any even permutation of the indices $1,2,3$ on the left-hand side induces a symmetry. For example, the transformation

$$
\begin{array}{ll}
x_{1} \rightarrow q x_{2}, & u_{1} \rightarrow u_{2}, \\
x_{2} \rightarrow x_{3}, & u_{2} \rightarrow u_{3}, \\
x_{3} \rightarrow x_{1}, & u_{3} \rightarrow u_{1},
\end{array}
$$

induced by the cyclic permutation (123), is a symmetry (it maps the generating function to $q^{u_{2}+u_{3}-u_{1}}$ times itself), as is the transformation

$$
x_{1} \rightarrow q x_{3}, \quad u_{1} \rightarrow u_{3},
$$

$$
\begin{aligned}
& x_{3} \rightarrow x_{2}, \quad u_{3} \rightarrow u_{2}, \\
& x_{2} \rightarrow x_{1}, \quad u_{2} \rightarrow u_{1},
\end{aligned}
$$

induced by (132), which maps the generating function to $q^{u_{3}+u_{1}-u_{2}}$ times itself. The odd permutation (12)(3) induces the transformation

$$
\begin{aligned}
& x_{1} \rightarrow x_{2}, \quad u_{1} \rightarrow u_{2}, \quad q \rightarrow q^{-1}, \\
& x_{2} \rightarrow x_{1}, \quad u_{2} \rightarrow u_{1}, \\
& x_{3} \rightarrow x_{3}, \quad u_{3} \rightarrow u_{3},
\end{aligned}
$$

which maps the generating function to

$$
(-1)^{u_{1}+u_{2}+u_{3}} q^{2\left(u_{1}^{2}+u_{2}^{2}+u_{3}^{2}\right)-(1 / 2)\left(u_{1}+u_{2}+u_{3}\right)^{2}+(3 / 2) u_{3}-(1 / 2) u_{1}-(1 / 2) u_{2}}
$$

times itself. The transformation

$$
x_{j} \rightarrow x_{j}^{-1}, \quad u_{j} \rightarrow u_{j}, \quad q \rightarrow q^{-1}, \quad j=1,2,3,
$$

followed by a multiplication by $x_{1}^{2 u_{1}} x_{2}^{2 u_{2}} x_{3}^{2 u_{3}}$, maps the generating function to

$$
(-1)^{u_{1}+u_{2}+u_{3}} q^{2\left(u_{1}^{2}+u_{2}^{2}+u_{3}^{2}\right)-1 / 2\left(u_{1}+u_{2}+u_{3}\right)^{2}+(3 / 2) u_{3}-(1 / 2) u_{1}-(1 / 2) u_{2}}
$$

times itself. The remaining symmetries are probably best understood from the examination of a new generating function. In (2.34) we set $x_{1}=z_{2} / y_{2}, x_{2}=z_{1} / y_{1}, x_{3}=z_{3} / y_{3}$, multiply by

$$
\frac{(-1)^{u_{1}+u_{2}+u_{3}}\left(y_{1} y_{3} s_{2}\right)^{u_{3}+u_{2}-u_{1}}\left(y_{2} y_{3} s_{1}\right)^{u_{3}+u_{1}-u_{2}}\left(y_{2} y_{1} s_{3}\right)^{u_{1}+u_{2}-u_{3}} q^{4\left(u_{1}^{2}+u_{2}^{2}+u_{3}^{2}\right)-\left(u_{1}+u_{2}+u_{3}\right)^{2}}}{(q ; q)_{u_{3}+u_{2}-u_{1}}(q ; q)_{u_{3}+u_{1}-u_{2}}(q ; q)_{u_{1}+u_{2}-u_{3}}},
$$

and sum over all possible values of $u_{1}, u_{2}, u_{3}$ to get

$\left(y_{3} s_{2} z_{1} q ; q\right)_{\infty}\left(y_{2} s_{1} z_{3} q ; q\right)_{\infty}\left(y_{1} s_{3} z_{2} q ; q\right)_{\infty}$

$\left(y_{1} s_{2} z_{3} ; q\right)_{\infty}\left(y_{3} s_{1} z_{2} ; q\right)_{\infty}\left(y_{2} s_{3} z_{1} q ; q\right)_{\infty}$

$$
\begin{aligned}
= & \sum_{u_{1}+u_{2}+u_{3}=0}^{\infty} \sum_{n_{j}=-u_{j}}^{u_{j}} \frac{s_{1}^{u_{3}+u_{1}-u_{2}} s_{2}^{u_{2}+u_{3}-u_{1}} s_{3}^{u_{1}+u_{2}-u_{3}}}{(q ; q)_{u_{3}+u_{1}-u_{2}}(q ; q)_{u_{2}+u_{3}-u_{1}}(q ; q)_{u_{1}+u_{2}-u_{3}}} z_{1}^{u_{2}+n_{2}} z_{2}^{u_{1}+n_{1}} z_{3}^{u_{3}-m} y_{1}^{u_{2}-n_{2}} y_{2}^{u_{1}-n_{1}}\left(-y_{3}\right)^{u_{3}+m} \\
& \times(-1)^{2\left(u_{2}+u_{3}\right)} q^{1 / 2\left(u_{1}+u_{2}-u_{3}\right)} \times q^{5 / 2\left(u_{1}^{2}+u_{2}^{2}+u_{3}^{2}\right)-2 u_{1} u_{2}-u_{3}\left(u_{1}+u_{2}\right)} q^{1 / 2\left[m\left(u_{2}-u_{1}+u_{3}\right)+n_{1}\left(u_{2}-u_{3}+u_{1}\right)+n_{2}\left(u_{3}-u_{1}+u_{2}+2\right)\right]}
\end{aligned}
$$




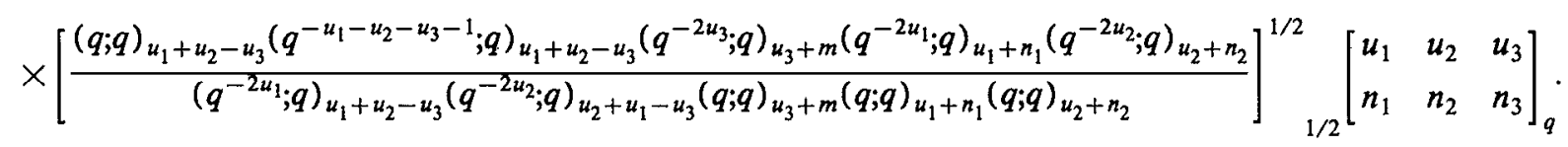

All even permutations of the rows and columns of the matrix,

$$
\left(\begin{array}{lll}
y_{1} & s_{1} & z_{1} \\
y_{2} & s_{2} & z_{2} \\
y_{3} & s_{3} & z_{3}
\end{array}\right)
$$

induce symmetries of the left-hand side of (2.35). For example, the mapping of (2.36) to

$$
\left(\begin{array}{lll}
y_{3} & z_{3} & s_{3} \\
y_{2} & z_{2} & s_{2} \\
y_{1} & z_{1} & s_{1}
\end{array}\right)
$$

is a symmetry, as is the mapping of (2.36) to

$$
\left(\begin{array}{ccc}
y_{3} & s_{3} & z_{3} \\
y_{1} & s_{1} & z_{1} q \\
y_{2} & s_{2} q^{-1} & z_{2}
\end{array}\right)
$$

and to

$$
\left(\begin{array}{ccc}
y_{2} & s_{2} & z_{2} q^{-1} \\
y_{3} & s_{3} q & z_{3} \\
y_{1} & s_{1} & z_{1}
\end{array}\right) .
$$

[The last two examples correspond to cyclic permutations of (2.34).] Another example is the mapping of (2.36) to

$$
\left(\begin{array}{ccc}
z_{1} q & y_{1} & s_{1} \\
z_{2} & y_{2} & s_{2} \\
z_{3} & y_{3} q^{-1} & s_{3}
\end{array}\right) .
$$

All these symmetries together generate the full group of 72 transformations of the Clebsch-Gordan coefficients. ${ }^{8}$ Through the relation (2.32) the symmetries lead to transformation formulas for the ${ }_{3} \phi_{2}$ polynomials. ${ }^{14}$

\section{A CLASS OF INFINITE-DIMENSIONAL REPRESENTATIONS OF $\mathrm{U}_{q}\left(\mathrm{su}_{2}\right)$}

Now we consider the discrete series $\uparrow_{u}$ of infinitedimensional representations of $\mathrm{U}_{q}\left(\mathrm{su}_{2}\right)$. This is defined on the Hilbert space $H_{0}$ with orthonormal basis $\left\{e_{m}: m=\right.$ $-u+n, n=0,1,2, \ldots\}$, such that

$$
E_{+} e_{m}=\left([m-u]_{q}[u+m+1]_{q}\right)^{1 / 2} e_{m+1},
$$

$$
\begin{aligned}
& E_{-} e_{m}=-\left([u+m]_{q}[m-u-1]_{q}\right)^{1 / 2} e_{m-1}, \\
& H e_{m}=m e_{m} .
\end{aligned}
$$

Here $u$ is a negative real number. A second convenient basis is the set $\left\{f_{n}: n=0,1, \ldots\right\}$, such that

$$
\begin{aligned}
& E_{+} f_{n}=q^{-1}[n-2 u]_{q} f_{n+1}, \\
& E_{-} f_{n}=-q[n]_{q} f_{n-1} \\
& H f_{n}=(-u+n) f_{n} .
\end{aligned}
$$

On this Hilbert space $E_{+}=-\left(E_{-}\right) *$ and $H^{*}=H$, i.e.,

$$
\left\langle E_{+} f, g\right\rangle=-\left\langle f, E_{-} g\right\rangle, \quad\langle H f, g\rangle=\langle f, H g\rangle,
$$

for all $f, g \in H_{0}$ in the domains of the appropriate operators $E_{ \pm}, H$. (In the limit as $q \rightarrow 1$ these representations correspond to the positive discrete series of unitary irreducible representations of the Lie algebra su( $(1,1) .^{5,6}$ ) Here

$$
f_{n}=\sqrt{\frac{q^{(3 / 2-u) n}(q ; q)_{n}}{\left(q^{-2 u} ; q\right)_{n}}} e_{-u+n}, \quad n=0,1,2, \ldots
$$

Note, however, that for each complex number $u$ such that $2 u \neq 0,1,2, \ldots$, expression ( 3.1$)$ (with a suitable definition of $\sqrt{[m-u]_{q}}$ ) or (3.2) defines an algebraically irreducible representation $\uparrow_{u}$ of $\mathrm{U}_{q}\left(\mathrm{su}_{2}\right)$ on an infinitedimensional vector space $K$, consisting of all finite linear combinations of the basis vectors $\left\{e_{m}\right\}$ or $\left\{\int_{n}\right\}$. In this more general case we can define a symmetric bilinear form $(\cdot, \cdot)$, such that $\left(e_{m}, e_{m^{\prime}}\right)=\delta_{m m^{\prime}}$. Then with respect to this form we have

$$
\left(E_{+} f, g\right)=-\left(f, E_{-} g\right),(H f, g)=(f, H g),
$$

for all $f, g \in K$. Also

$$
\left(f_{n} f_{n^{\prime}}\right)=\delta_{n n^{\prime}} \frac{q^{(3 / 2-u) n}(q ; q)_{n}}{\left(q^{-2 u} ; q\right)_{n}}
$$

Given the irreducible representations $\uparrow_{u_{1}}$ and $\uparrow_{u_{2}}$ we define the tensor product representation $\uparrow_{u_{1}} \otimes{ }_{a} \uparrow_{u_{2}}$ of $\mathrm{U}_{q}\left(\mathrm{su}_{2}\right)$ on the space $K \otimes K$ by the usual operators 


$$
\begin{aligned}
& F_{+}=\Delta_{a}\left(E_{+}\right)=E_{+} \otimes q^{a H}+q^{(a-1) H} \otimes E_{+}, \\
& F_{-}=\Delta_{a}\left(E_{-}\right)=E_{-} \otimes q^{(1-a) H}+q^{-a H} \otimes E_{-}, \\
& L=\Delta_{a}(H)=H \otimes I+I \otimes H,
\end{aligned}
$$

where $a$ is a complex number. Again the operators $F_{ \pm}, L$ satisfy the same commutation relations as the operators $E_{ \pm}, H$. [If $u_{1}$ and $u_{2}$ are negative real numbers and we require that $F_{-}=\left(F_{+}\right)^{*}, L=L^{*}$ with respect to the inner product induced from the unitary representations $\uparrow_{u_{1}}$, $\dagger_{u_{2}}$; then we must have $a=\frac{1}{2}$. Since the equivalence relations $(2.10)$ hold, we can relate the tensor product $\Delta_{a}$ for general $a$ to $\Delta_{1 / 2}$.]

To decompose $\uparrow_{u_{1}} \otimes{ }_{a} \uparrow_{u_{2}}$ into a direct sum of irreducible representations we follow the procedure of Sec. II and introduce a convenient one-variable model of $\uparrow_{u}{ }^{11} \mathrm{~A}$ basis for the vector space consists of the functions $\left\{f_{n}(z)\right.$ $\left.=z^{n}: n=0,1,2, \ldots\right\}$ in the complex variable $z$. The action of $\mathrm{U}_{q}\left(\mathrm{su}_{2}\right)$ on functions $f(z)$ is defined by expressions (2.11). We define a bilinear form $(\cdot, \cdot)$, such that

$$
(f, g)=\frac{1}{2 \pi} \int_{0}^{2 \pi} \int_{0}^{k} f\left(r e^{i \theta}\right) g\left(r e^{-i \theta}\right) \rho\left(r^{2}\right) d r^{r^{2}} d \theta,
$$

where $z=r e^{i \theta}, \bar{z}=r e^{-i \theta}$, and

$$
\int_{0}^{k} F\left(r^{2}\right) d_{q} r^{2}=k(1-q) \sum_{n=0}^{\infty} F\left(k q^{n}\right) q^{n}
$$

\section{Requiring that}

$$
\left(E_{+} f, g\right)=-\left(f, E_{-} g\right), \quad(H f, g)=(f, H g),
$$

for all polynomials $f, g$ we find that the (essentially) unique solution is

$$
k=q^{-u+1 / 2}, \quad \rho\left(r^{2}\right)=\frac{\left[u-\frac{1}{2}\right]_{q}\left(r^{2} q^{u-1 / 2} ; q\right)_{\infty}}{q^{5 / 2}\left(r^{2} q^{-u-5 / 2} ; q\right)_{\infty}},
$$

for $u \neq-\frac{1}{2}$, normalized so that $\|1\|^{2}=\langle 1,1\rangle=1$. In the special case $u=-\frac{1}{2}$ we define the bilinear form through the limit

$$
(f, g)_{-1 / 2}=\lim _{u \rightarrow-1 / 2}(f, g)_{u} .
$$

If $u$ is real and negative this bilinear form determines an inner product $\langle f, g\rangle=(f, \bar{g})$. With respect to this inner product we have relations

$$
\left\langle f_{n}, f_{n^{\prime}}\right\rangle=\delta_{n n^{\prime}} \frac{q^{(3 / 2-u) n}(q ; q)_{n}}{.\left(q^{-2 u} ; q\right)_{n}},
$$

in agreement with (3.6). Completing the vector space $K$ to the Hilbert space $K^{u}$, the closure of $K$ with respect to this inner product, we see that $K_{u}$ consists of all functions

$$
f(z)=\sum_{n=0}^{\infty} c_{n} z^{n}
$$

such that

$$
\sum_{n=0}^{\infty}\left|c_{n}\right|^{2} \frac{\left(q^{3 / 2-u}\right)^{n}(q ; q)_{n}}{\left(q^{-2 u} ; q\right)_{n}}<\infty
$$

These are functions $f(z)$ analytic in the disk $|z|<q^{u / 2-3 /}$ 4. The Hilbert spaces $K_{u}$ have corresponding kernel functions

$$
S\left(\bar{z}^{\prime}, z\right)=\sum_{n=0}^{\infty} e_{-u+n}\left(\bar{z}^{\prime}\right) e_{-u+n}(z)=\frac{\left(q^{-u-3 / 2} \bar{z}^{\prime} z ; q\right)_{\infty}}{\left(q^{u-3 / 2} \bar{z}^{\prime} z ; q\right)_{\infty}},
$$

so that

$$
\left(g, S\left(\bar{z}^{\prime}, \cdot\right)=g\left(z^{\prime}\right),\right.
$$

for $\left|z^{\prime}\right|<q^{u / 2-3 / 4}$ and $g \in K_{u}$.

Just as in Sec. II, the operators corresponding to the tensor product $\uparrow_{u_{1}} \otimes{ }_{a} \uparrow_{u_{2}}$ take the form (2.15). The functions

$$
p_{k, l}(z, w)=z^{k} w^{l}, \quad k, l=0,1,2, \ldots,
$$

form an orthogonal basis for $K_{u_{1}} \otimes K_{u_{2}}$. Again we will use the weight vector calculations to decompose $K_{u_{1}} \otimes K_{u_{2}}$ into irreducible subspaces. For convenience, we will consider only the case where $u_{1}$ and $u_{2}$ are negative real numbers. [However, it is easy, via the bilinear form (3.8), to carry out the corresponding computation for all complex $u_{1}, u_{2}$ such that $2 u_{1}, 2 u_{2}$ and $2\left(u_{1}+u_{2}\right)$ are not positive integers or zero.] The eigenvectors $f$ of $L$ such that $F_{-} f=0$ are given by the expression (2.16), where now $s=0,1,2, \ldots$. In the case where $a=\frac{1}{2}$ we can sum this series explicitly:

$$
\begin{aligned}
f_{s, 0} & =z^{s} \frac{\left((w / z) q^{\left.-(1 / 2) u_{1}-(1 / 2) u_{2} ; q\right)_{\infty}}\right.}{\left((w / z) q^{-(1 / 2) u_{1}-(1 / 2) u_{2}+s} ; q\right)_{\infty}} \\
& =z^{s}\left(\frac{w}{z} q^{-(1 / 2) u_{1}-(1 / 2) u_{2} ; q}\right)_{s} \\
L f_{s, 0} & =\left(s-u_{1}-u_{2}\right) f_{s, 0}, \quad s=0,1, \ldots .
\end{aligned}
$$

Now we introduce a bilinear form $\langle\cdot, \cdot\rangle_{a}$ on $K_{u_{1}} \otimes K_{u_{2}}$, such that 


$$
\begin{aligned}
\left\langle p_{k_{1}, l_{1}}, p_{k_{2}, l_{2}}\right\rangle_{a}= & \delta_{k_{1} k_{2}} \delta_{l_{1} l_{2}} q^{(3 / 2)\left(k_{1}+l_{1}\right)-u_{1} k_{1}-u_{2} l_{1}} \\
& \times q^{(2 a-1)\left(u_{1} l_{1}+u_{2} k_{2}-k_{1} l_{1}\right)} \\
& \times \frac{(q ; q)_{k_{1}}(q ; q)_{l_{1}}}{\left(q^{\left.-2 u_{1} ; q\right)_{k_{1}}\left(q^{\left.-2 u_{2} ; q\right)_{l_{1}}}\right.}\right.}
\end{aligned}
$$

It is easy to verify that

$$
\left\langle F_{+} p_{1}, p_{2}\right\rangle_{a}=-\left\langle p_{1}, F_{-} p_{2}\right\rangle_{a}, \quad\left\langle L p_{1}, p_{2}\right\rangle=\left\langle p_{1}, L p_{2}\right\rangle_{a}
$$

for all $p_{1}, p_{2} \in K_{u_{1}} \otimes K_{u_{2}}$. [For $a=\frac{1}{2}$, this agrees with the inner product induced on $K_{u_{1}} \otimes K_{u_{2}}$ by (3.10) and (3.12).] It follows that

$$
\begin{aligned}
\left\langle f_{s, 0}^{a}, f_{s, 0}^{a}\right\rangle_{a}= & q^{-u_{1} s+(2 a-1) u_{2} s+(3 / 2) s} \\
& \times \frac{(q ; q)_{s}\left(q^{-2 u_{2}-2 u_{1}+s-1} ; q\right)_{s}}{\left(q^{-2 u_{1}} ; q\right)_{s}\left(q^{-2 u_{2}} ; q\right)_{s}} .
\end{aligned}
$$

Vectors $f_{s, k}$ can now be defined, recursively, by

$$
f_{s, k+1}=\frac{-q}{\left[2 u_{1}+2 u_{2}-2 s-k\right]_{q}} F_{+} f_{s, k} \quad s, k=0,1, \ldots
$$

\section{Lemma 5:}

$$
\begin{gathered}
\text { (1) } F_{+} f_{s, k}=-q^{-1}\left[2 u_{1}+2 u_{2}-2 s-k\right]_{q} f_{s, k+1}, \\
\text { (2) } F_{-} f_{s, k}=-q[k]_{q} f_{s, k-1}, \\
\text { (3) } L f_{s, k}=\left(-u_{1}-u_{2}+s+k\right) f_{s, k}, \\
s, k=0,1 \ldots
\end{gathered}
$$

For fixed $s$ the $\left\{f_{s, k}\right\}$ form an orthogonal basis for a subspace of $K_{u_{1}} \otimes K_{u_{2}}$ transforming according to the irreducible representation $\uparrow u_{1}+u_{2}-s$.

\section{Lemma 6:}

$$
\uparrow_{u_{1}} \otimes_{a} \uparrow_{u_{2}} \cong \sum_{s=0}^{\infty} \oplus \uparrow_{u_{1}+u_{2}-s}
$$

Lemma 7:

$$
\begin{aligned}
\left\langle f_{s, k}^{a} f_{s^{\prime}, k^{\prime}}^{a}\right\rangle & \\
= & \left.\delta_{s s^{\prime}} \delta_{k k^{\prime}} q^{u_{1} s+(2 a-1) u_{2} s+(3 / 2) s+k\left(-u_{1}-u_{2}+s+3 / 2\right.}\right) \\
& \times \frac{(q ; q)_{k}(q ; q)_{s}\left(q^{-2 u_{1}-2 u_{2}+s-1} ; q\right)_{s}}{\left(q^{-2 u_{1}} ; q\right)_{s}\left(q^{-2 u_{2}} ; q\right)_{s}\left(q^{-2 u_{1}-2 u_{2}+2 s} ; q\right)_{k}} .
\end{aligned}
$$

Passing to the orthonormal basis $\left\{e_{m}^{v}\right\}$, where

$$
\begin{aligned}
& e_{m}^{v}=\left\|f_{s, k}\right\|^{-1} f_{s, k}, \\
& v=u_{1}+u_{2}-s, \quad m=-u_{1}-u_{2}+s+k,
\end{aligned}
$$

we see that these basis vectors satisfy relations (3.1).

The derivation of a generating function for the Clebsch-Gordan coefficients is very similar to the corresponding computation in Sec. II. We apply a $q$ analog of the exponential $F_{+}$to $f_{s, 0}^{a}$, exactly as in (2.23). Applying Lemma 4 and using (2.16) we again obtain (2.25). Similarly, the generating function (2.26) and the explicit expression (2.27) for $f_{s, k}^{a}(z, w)$ hold for the discrete series of representations. From this result we can expand the orthonormal basis $\left\{e_{m}^{v}\right\}$ for $K_{u_{1}} \otimes K_{u_{2}}$,

$$
f_{s, k}^{a}=\left[\frac{(q ; q)_{u_{1}+u_{2}-v}\left(q^{-u_{1}-u_{2}-v-1} ; q\right)_{u_{1}+u_{2}-v}(q ; q)_{v+m}}{\left(q^{-2 u_{1}} ; q\right)_{u_{1}+u_{2}-v}\left(q^{-2 u_{2}} ; q\right)_{u_{1}+u_{2}-v}\left(q^{-2 v} ; q\right)_{m+v}}\right]^{1 / 2} q^{(1 / 2)\left(u_{1}+u_{2}-v\right)\left(-u_{1}+[2 a-1] u_{2}+3 / 2\right)+(1 / 2)(m+v)(3 / 2-v)} e_{m}^{v}
$$

where

$$
m=s+k-u_{1}-u_{2}, \quad v=u_{1}+u_{2}-s,
$$

in terms of the orthonormal basis

$$
\begin{aligned}
e_{n_{1}}^{u_{1}} \otimes_{a} e_{n_{2}}^{u_{2}}= & {\left[\frac{\left(q^{-2 u_{1}} ; q\right)_{u_{1}+n_{1}}\left(q^{-2 u_{2}} ; q\right)_{u_{2}+n_{2}}}{(q ; q)_{u_{1}+n_{1}}(q ; q)_{u_{2}+n_{2}}}\right]^{1 / 2} q^{(1 / 2-a)\left[u_{1}\left(u_{1}+n_{1}\right)+u_{2}\left(u_{2}+n_{2}\right)-\left(u_{1}+n_{1}\right)\left(u_{2}+n_{2}\right)\right]} } \\
& \times q^{-(3 / 4)\left(u_{1}+u_{2}+n_{1}+n_{2}\right)+\left(u_{1} / 2\right)\left(u_{1}+n_{1}\right)+\left(u_{2} / 2\right)\left(u_{2}+n_{2}\right)} p_{u_{1}+n_{1}, u_{2}+n_{2}}, \quad n_{i}=-u_{i}-u_{i}+1, \ldots:
\end{aligned}
$$


$e_{m}^{v}=\sum_{n_{1}, n_{2}}\left[\begin{array}{lll}u_{1} & u_{2} & v \\ n_{1} & n_{2} & m\end{array}\right]_{q} e_{n_{1}}^{u_{1}} \otimes d_{n_{2}}^{u_{2}}$

This last expression defines the Clebsch-Gordan coeffi- cients for the tensor product $\uparrow_{u_{1}} \otimes \uparrow_{u_{2}}$. Clearly, they vanish unless $m=n_{1}+n_{2}$. The orthogonality of the two bases implies the orthogonality relations (2.31), except that the sums are now infinite. We find

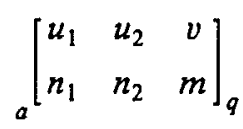

$$
\begin{aligned}
& =\left[\frac{\left(q^{-2 u_{2}} ; q\right)_{u_{1}+u_{2}-v}\left(q^{-2 u_{1}} ; q\right)_{u_{1}+n_{1}}\left(q^{-2 u_{2}} ; q\right)_{u_{2}+n_{2}}(q ; q)_{v+m}(q ; q)_{u_{1}+n_{1}}(q ; q)_{u_{2}+n_{2}}}{\left(q^{-u_{1}-u_{2}-v-1} ; q\right)_{u_{1}+u_{2}-v}\left(q^{-2 u_{1}} ; q\right)_{u_{1}+u_{2}-v}\left(q^{-2 v} ; q\right)_{v+m}}\right]^{1 / 2} \\
& \times\left(q^{v-u_{2}+n_{1}+1} ; q\right)_{\infty}\left(q^{u_{2}+n_{2}+1} ; q\right)_{\infty} q^{-(1 / 2)\left(u_{1}+u_{2}-v\right)\left(-u_{1}+[1-2 a] n_{2}+3 / 2\right)+(m+v)\left((1 / 2) u_{1}-u_{2}+[a-1 / 2] n_{2}-3 / 4\right)} \\
& \times q^{(a-1 / 2)\left[u_{1}\left(u_{1}+n_{1}\right)+u_{2}^{2}-n_{2}^{2}-\left(u_{1}+n_{1}\right)\left(u_{2}+n_{2}\right)\right]} q^{-\left(u_{1} / 2\right)\left(u_{1}+n_{1}\right)+u_{2}\left(u_{2}+n_{2}\right)-a u_{1}\left(u_{2}+n_{2}\right)} \\
& \times q^{(3 / 4)\left(u_{1}+u_{2}+n_{1}+n_{2}\right)}{ }_{3} \phi_{2}\left(\begin{array}{ccc}
q^{v-u_{1}-u_{2}}, & q^{u_{1}-u_{2}+v+1}, & q^{-u_{2}-n_{2}} \\
q^{v+n_{1}-u_{2}+1}, & q^{-2 u_{2}} & ; q ; q^{-v+m}
\end{array}\right) .
\end{aligned}
$$

Finally, in the case $a=\frac{1}{2}$ the sum (2.25) can be evaluated explicitly to yield the generating function

$$
\left(\exp _{q} t F_{+}\right) f_{s, 0}^{\mathrm{l} / 2}=\frac{\left([z t /(1-q)] q^{u_{1} / 2-v / 2} ; q\right)_{\infty}\left([w t /(1-q)] q^{u_{1}-u_{2} / 2-v / 2} ; q\right)_{\infty}\left((w / z) q^{-u_{1} / 2-u_{2} / 2} ; q\right)_{u_{1}+u_{2}-v}}{\left([w t /(1-q)] q^{u_{2} / 2+v / 2} ; q\right)_{\infty}\left([z t /(1-q)] q^{u_{1} / 2-u_{2}+v / 2} ; q_{\infty}\right.}
$$

\section{MODELS OF OSCILLATOR ALGEBRA REPRESENTATIONS}

We introduce as a $q$ analog of the oscillator algebra the associative algebra generated by the four elements $H$, $E_{+}, E_{-}, \mathscr{C}$ that obey the commutation relations

$$
\begin{aligned}
& {\left[H, E_{+}\right]=E_{+}, \quad\left[H, E_{-}\right]=-E_{-},} \\
& {\left[E_{+}, E_{-}\right]=-q^{-H \mathscr{E},}\left[\mathscr{E}, E_{ \pm}\right]=[\mathscr{E}, H]=0 .}
\end{aligned}
$$

These relations are motivated by the recurrence relations obeyed by the $q$-Laguerre polynomials, although, as we shall see, this associative algebra is not a quantum algebra. In the limit as $q \rightarrow 1$, expressions (4.1) reduce to the commutation relations of the four-dimensional oscillator Lie algebra. ${ }^{1}$ The associative algebra admits a class of algebraically irreducible representations $\uparrow_{l, \lambda}$, where $l, \lambda$ are complex numbers and $l \neq 0$. These are defined on a vector space with basis $\left\{e_{n}: n=0,1,2, \ldots\right\}$, such that

$$
\begin{aligned}
& E_{+} e_{n}=l \sqrt{\frac{q^{-n-1}-1}{1-q}} e_{n+1}, \\
& E_{-} e_{n}=l \sqrt{\frac{q^{-n}-1}{1-q}} e_{n-1},
\end{aligned}
$$

$$
H e_{n}=(\lambda+n) e_{n}, \quad \mathscr{C} e_{n}=l^{2} q^{\lambda-1} e_{n}
$$

If $\lambda$ and $l$ are real, then $\uparrow_{l, \lambda}$ is defined on the Hilbert space $K_{0}$ with orthonormal basis $\left\{e_{n}\right\}$, and on this space we have $E_{+}=\left(E_{-}\right)^{*}, H^{*}=H$ and $\mathscr{C} *=\mathscr{C}$. A second convenient basis for $K_{0}$ is $\left\{f_{n}: n=0,1, \ldots\right\}$, where

$$
\begin{aligned}
& E_{+} f_{n}=l q^{-(n+1) / 2} f_{n+1}, \\
& E_{-} f_{n}=l q^{-n / 2} \frac{1-q^{n}}{1-q} f_{n-1}, \\
& H f_{n}=(\lambda+n) f_{n}, \quad \mathscr{E} f_{n}=l^{2} q^{\lambda-1} f_{n} .
\end{aligned}
$$

Here, $f_{n}=\sqrt{(q ; q)_{n} /(1-q)^{n}} e_{n}$.

Note that even in the case where $l$ and $\lambda$ are complex, we can define a symmetric bilinear form $(\cdot, \cdot)$ on the space $K$ of all finite linear combinations of the basis vectors $\left\{e_{n}\right\}$, such that $\left(e_{n}, e_{n^{\prime}}\right)=\delta_{n n^{\prime}}$. Then, with respect to this bilinear form, we have

$$
\begin{aligned}
& \left(E_{+} f, g\right)=\left(f, E_{-} g\right), \quad(H f, g)=(f, H g), \\
& (\mathscr{E} f, g)=(f, \mathscr{C} g),
\end{aligned}
$$

for all polynomials $f, g \in K$. Also, 


$$
\left(f_{n}, f_{n}^{\prime}\right)=\delta_{n n^{\prime}} \frac{(q ; q)_{n}}{(1-q)^{n}}
$$

The elements $\mathscr{C}=q q^{-H} \mathscr{C}+(q-1) E_{+} \mathrm{E}_{-}$and $\mathscr{E}$ lie in the center of this algebra, and corresponding to the irreducible representation $\uparrow_{l, \lambda}$ we have $\mathscr{C}=l^{2} I, \mathscr{C}=l^{2} q^{\lambda-1} I$, where $I$ is the identity operator on $K_{0}$.

Given the irreducible representations $\uparrow_{l,}, \lambda_{1}$ and $\uparrow_{l_{2}, \lambda_{2}}$ on the Hilbert space $K_{0}$ we define the tensor product representation $\uparrow_{l_{1}, \lambda_{1}} \otimes{ }_{a} \uparrow_{l_{2}, \lambda_{2}}$ on the space $K_{0} \otimes K_{0}$ by the operators

$F_{+}=\Delta_{a}\left(E_{+}\right)=E_{+} \otimes q^{a H}+q^{(a-1) H} \otimes E_{+}$,

$F_{-}=\Delta_{a}\left(E_{-}\right)=E_{-} \otimes q^{(1-a) H}+q^{-a H} \otimes E_{-}\left(\kappa_{1} q^{H}+\kappa_{2}\right)$

$L=\Delta_{a}(H)=H \otimes I+I \otimes H$,

$\mathscr{F}=\Delta_{a}(\mathscr{C})=\mathscr{C} \otimes I+I \otimes \mathscr{C}=\left(l_{1}^{2} q^{\lambda_{1}-1}+l_{2}^{2} q^{\lambda_{2}-1}\right) I \otimes I$,

where

$$
\kappa_{1}=-\frac{l_{1}^{2} q^{\lambda_{1}-1}}{l_{2}^{2}}, \quad \kappa_{2}=\frac{l_{1}^{2} q^{\lambda_{1}}+l_{2}^{2} q^{\lambda_{2}}}{l_{2}^{2} q^{\lambda_{2}}} .
$$

Then we have

$$
\begin{aligned}
& {\left[L, F_{ \pm}\right]= \pm F_{ \pm}, \quad\left[F_{+}, F_{-}\right]=-\mathscr{F} q^{-L},} \\
& {\left[\mathscr{F}, F_{ \pm}\right]=[\mathscr{F}, L]=0,}
\end{aligned}
$$

in agreement with (4.1). Here $a$ is a complex constant.

A second type of tensor product representation $\uparrow_{l_{1}, \lambda_{1}} \otimes{ }_{a}^{\prime} \uparrow_{l_{2}, \lambda_{2}}$ is defined on $K_{0} \otimes K_{0}$ by the operators

$F_{+}^{\prime}=\Delta_{a}^{\prime}\left(E_{+}\right)=E_{+} \otimes q^{a H}+q^{(a-1) H} \otimes E_{+}\left(\xi_{1} q^{1 / 2 H}+\xi_{2}\right)$,

$F_{-}^{\prime}=\Delta_{a}^{\prime}\left(E_{-}\right)=E_{-} \otimes q^{(1-a) H}+q^{-a H} \otimes E_{-}\left(\kappa_{1} q^{1 / 2 H}+\kappa_{2}\right)$

$L^{\prime}=\Delta_{a}^{\prime}(H)=H \otimes I+I \otimes H$,

$$
\mathscr{F}^{\prime}=\Delta_{a}^{\prime}(\mathscr{E})=\mathscr{C} \otimes I+I \otimes \mathscr{C}=\left(l_{1}^{2} q^{\lambda_{1}-1}+l_{2}^{2} q^{\lambda_{2}-1}\right) I \otimes I,
$$

where

$\xi_{1}=-\frac{\tau l_{1}^{2} q^{\lambda_{1}-1 / 2}}{l_{2}^{2}}, \quad \xi_{2}=-\frac{\tau l_{1} q^{\lambda_{1} / 2}}{l_{2}^{2} q^{\lambda_{2} / 2}} \sqrt{l_{1}^{2} q^{\lambda_{1}-1}+l_{2}^{2} q^{\lambda_{2}-1}}$,

$\kappa_{1}=\frac{1}{\tau}, \quad \kappa_{2}=-\frac{q^{1 / 2} \sqrt{l_{1}^{2} q^{\lambda_{1}}+l_{2}^{2} q^{\lambda_{2}}}}{\tau l_{1} q^{\left(\lambda_{1}+\lambda_{2}\right) / 2}}$.
Here $a$ and $\tau$ are complex constants. Neither of these coproducts leads to a quantum algebra because, for example neither satisfies the associative law. ${ }^{15}$

Since relations (2.10) hold, the operators $\Delta_{a}, \Delta_{a}^{\prime}$ are equivalent to the corresponding operators $\Delta_{1 / 2}, \Delta_{1 / 2}^{\prime}$. Using this equivalence we shall assume $a=\frac{1}{2}$ in the computations to follow.

We introduce two convenient one-variable models of $\uparrow_{\ell, \lambda}$. In the first case a basis for the vector space consists of the functions $\left\{f_{n}(z)=z^{n}: n=0,1,2, \ldots\right\}$ in the complex variable $z$. The action of the oscillator algebra is given by the operators

$$
\begin{aligned}
& E_{+}=\frac{l z}{q^{1 / 2}} T_{z}^{-1 / 2}, \quad E_{-}=\frac{l}{(1-q) z}\left(T_{z}^{-1 / 2}-T_{z}^{1 / 2}\right), \\
& H=\lambda+z \frac{d}{d z}, \quad \mathscr{B}=l^{2} q^{\lambda-1} I
\end{aligned}
$$

where $T_{z}^{a} f(z)=f\left(q^{\alpha} z\right)$. Thus relations (4.3) hold. We define a bilinear form (3.8) and (3.9), such that

$$
\begin{aligned}
& \left(E_{+} f, g\right)=\left(f, E_{-} g\right), \quad(H f, g)=(f, H g), \\
& (\mathscr{E} f, g)=(f, \mathscr{E} g),
\end{aligned}
$$

for all polynomials $f, g$. The essentially unique solution is

$$
k=\frac{1}{1-q}, \quad \rho\left(r^{2}\right)=\left(q(1-q) r^{2} ; q\right)_{\infty} ;
$$

see Ref. 2. For $l$ and $\lambda$ real the bilinear form induces an inner product $\langle\cdot, \cdot\rangle$, such that

$$
\begin{aligned}
& \left.\left\langle E_{+} f, g\right\rangle=\left\langle f, E_{-} g\right\rangle, \quad\langle H f, g)\right\rangle=\langle f, H g\rangle, \\
& \langle\mathscr{E} f, g\rangle=\langle f, \mathscr{E} g\rangle,
\end{aligned}
$$

for all polynomials $f$ and $g$. The functions

$$
e_{n}=\sqrt{\frac{(1-q)^{n}}{(q ; q)_{n}}} z^{n}, \quad n=0,1, \ldots
$$

form an orthonormal basis for the Hilbert space $K_{0}$ of all functions

$$
f(z)=\sum_{n=0}^{\infty} c_{n} z^{n}
$$

such that

$$
\sum_{n=0}^{\infty} \frac{\left|c_{n}\right|^{2}}{(1-q)^{n}}<\infty
$$


It follows that these functions are analytic in the disk $|z|<(1-q)^{-1 / 2}$. The Hilbert space $K_{0}$ has the kernel function

$$
S\left(\bar{z}^{\prime}, z\right)=\sum_{n=0}^{\infty} e_{n}\left(\overline{z^{\prime}}\right) e_{n}(z)=\frac{1}{\left((1-q) \bar{z}^{\prime} z ; q\right)_{\infty}},
$$

so that

$$
\left\langle g, S\left(\vec{z}^{\prime}, \cdot\right\rangle=g\left(z^{\prime}\right),\right.
$$

for $\left|z^{\prime}\right|<(1-q)^{-1 / 2}$ and $f \in K_{0}$.

A second model of $\uparrow, 1,11$ is determined by the basis functions $\left\{f_{n}(z)=q^{n(n+1) / 4} z^{n}: n=0,1,2, \ldots\right\}$, and the operators

$$
\begin{aligned}
& E_{+}=l z I, \quad E_{-}=\frac{l}{(1-q) z}\left(1-T_{z}^{-1}\right) \\
& H=\lambda+z \frac{d}{d z}, \quad \mathscr{E}=l^{2} q^{\lambda-1} I .
\end{aligned}
$$

Then expressions (4.3) are valid. We define a bilinear form, such that

$$
\left(f_{, g}\right)=\iint_{-\infty}^{\infty} f(z) g(\bar{z}) \rho(z, \bar{z}) d x d y
$$

where $z=x+i y$ and $f, g$ are polynomials in $z$. Requiring that conditions (4.12) hold, we obtain the solution

$$
\rho(z, \bar{z})=\frac{1-q}{(-(1-q) z \bar{z} ; q)_{\infty} \pi \ln q^{-1}} .
$$

For $l$ and $\lambda$ real this bilinear form induces an inner product $\langle\cdot, \cdot\rangle$ for $K_{0}$, such that relations (4.14) hold for all polynomials $f$ and $g$. The functions

$$
e_{n}^{\prime}=q^{n(n+1) / 4} \sqrt{\frac{(1-q)^{n}}{(q ; q)_{n}}} z^{n}, \quad n=0,1, \ldots,
$$

form an orthonormal basis for the Hilbert space $K_{0}$ of all functions,

$$
f^{\prime}(z)=\sum_{n=0}^{\infty} c_{n} z^{n}
$$

such that

$$
\sum_{n=0}^{\infty} \frac{\left|c_{n}\right|^{2} q^{-n(n+1) / 2}}{(1-q)^{n}}<\infty .
$$

This is a space of entire functions; it has the kernel function
$S\left(\bar{z}^{\prime}, z\right)=\sum_{n=0}^{\infty} e_{n}^{\prime}\left(\bar{z}^{\prime}\right) e_{n}^{\prime}(z)=\left(-(1-q) q \bar{z}^{\prime} z ; q\right)_{\infty}$

\section{TENSOR PRODUCTS OF OSCILLATOR REPRESENTATIONS}

We will make use of the model (4.11) of the oscillator algebra to decompose the representation $\uparrow_{l_{1}, \lambda_{1}}$ $\otimes \uparrow_{l_{2}, \lambda_{2}}$ into irreducible components. Thus we have

$$
F_{+}=\frac{l_{1} z}{q^{1 / 2}} T_{z}^{-1 / 2} q^{\lambda_{2} / 2} T_{w}^{1 / 2}+\frac{l_{2} w}{q^{1 / 2}} T_{w}^{-1 / 2} q^{-\lambda_{1} / 2} T_{z}^{-1 / 2}
$$

$$
\begin{aligned}
F_{-}= & \frac{l_{1}}{(1-q) z}\left(T_{z}^{-1 / 2}-T_{z}^{1 / 2}\right) q^{\lambda_{2} / 2} T_{w}^{1 / 2} \\
& +q^{-\lambda_{1} / 2} T_{z}^{-1 / 2} \frac{l_{2}}{(1-q) w}\left(T_{w}^{-1 / 2}-T_{w}^{1 / 2}\right) \\
& \times\left(\kappa_{1} q^{\lambda_{2}} T_{w}+\kappa_{2}\right),
\end{aligned}
$$

$L=\lambda_{1}+\lambda_{2}+z \frac{d}{d z}+w \frac{d}{d w}$,

$\mathscr{F}=l_{1}^{2} q^{\lambda_{1}-1}+l_{2}^{2} q^{\lambda_{2}-1}$,

where

$$
\kappa_{1}=-\frac{l_{1}^{2} q^{\lambda_{1}-1}}{l_{2}^{2}}, \quad \kappa_{2}=\frac{l_{1}^{2} q^{\lambda_{1}}+l_{2}^{2} q^{\lambda_{2}}}{l_{2}^{2} q^{\lambda_{2}}}
$$

The functions

$$
p_{k_{1}, k_{2}}(z, w)=z^{k_{1}} w^{k_{2}}, \quad k_{1}, k_{2}=0,1, \ldots
$$

form a basis for $K_{0} \otimes K_{0}$. The eigenvectors $f$ of $L$, such that $F_{-} f=0$, are given by

$$
\begin{aligned}
f_{s, 0}(z, w)= & z^{s} \sum_{k=0}^{s} \frac{\left(q^{-s} ; q\right)_{k}}{(q ; q)_{k}\left(-\left(\kappa_{1} / \kappa_{2}\right) q^{\lambda_{2}+1} ; q\right)_{k}} \\
& \times\left[\frac{w l_{1} q^{\left(\lambda_{1}+\lambda_{2}+3 s\right) / 2}}{z l_{2} \kappa_{2}}\right]^{k} .
\end{aligned}
$$

Using (2.23) and Lemma 4, we find 


$$
\begin{aligned}
\left(\exp _{q} t F_{+}\right) f_{s, 0}= & \sum_{h=0}^{s} \sum_{j, l=0}^{\infty} \frac{\left(q^{-s} ; q\right)_{h} q^{h j}}{\left(-\left(\kappa_{1} / \kappa_{2}\right) q^{\lambda_{2}+1} ; q\right)_{h}(q ; q)_{h}(q ; q)_{j}(q ; q)_{l}}\left(q^{\lambda_{2} / 2-s / 2} l_{1}\right)^{j}\left(q^{-\lambda_{1} / 2-s / 2} l_{2}\right)^{l} \\
& \times\left(\frac{q^{\lambda_{1} / 2+\lambda_{2} / 2+s} l_{1}}{l_{2} \kappa_{2}}\right)^{h} z^{s-h+j} w^{h+l_{t} j+l} \\
= & \frac{z^{s}}{\left(q^{-\lambda_{1} / 2-s / 2} l_{2} t w ; q\right)_{\infty}\left(q^{\lambda_{2} / 2-s / 2} l_{1} t z ; q\right)_{\infty}}{ }_{2} \phi_{1}\left(-\frac{\kappa_{1}}{\kappa_{2}} q^{\lambda_{2}+1} \quad ; \quad ; \quad \frac{q^{\lambda_{2} / 2-s / 2} l_{1} t z}{l_{2} \kappa_{2} z}\right) .
\end{aligned}
$$

We introduce a bilinear form $\{\cdot, \cdot\}$ on $K_{0} \otimes K_{0}$, such that

$\left\langle p_{h, j} p_{h^{\prime}, j^{\prime}}\right\rangle=\delta_{h h^{\prime}} \delta_{j j^{\prime}} \frac{\left(-\left(\kappa_{1} / \kappa_{2}\right) q^{\lambda_{2}+1} ; q\right)_{j}}{(1-q)^{h+j}}(q ; q)_{h}(q ; q) \mu_{j}^{j}$

Then we have

$$
\left\langle F_{+} p_{1} p_{2}\right\rangle=\left\langle p_{1}, F_{-} p_{2}\right\rangle, \quad\left\langle L p_{1}, p_{2}\right\rangle=\left\langle p_{1}, L p_{2}\right\rangle,
$$

for all $p_{1}, p_{2} \in K_{0} \otimes K_{0}$.

Defining the functions $f_{s, k}(z, w)$, recursively, by

$$
\begin{aligned}
& f_{s, k+1}=\tilde{l}_{s}^{-1} q^{(k+1) / 2} F_{+} f_{s, k}, \quad s, k=0,1, \ldots, \\
& \tilde{l}_{s}=\sqrt{q^{-s}\left(l_{1}^{2} q^{-\lambda_{2}}+l_{2}^{2} q^{-\lambda_{1}}\right)},
\end{aligned}
$$

we obtain the following.

Lemma 8:

(1) $F_{+} f_{s, k}=\tilde{l}_{s} q^{-(k+1) / 2} f_{s, k+1}$,

(2) $F_{-} f_{s, k}=\tilde{l}_{s} q^{-k / 2}\left[\left(1-q^{k}\right) /(1-q)\right] f_{s, k-1}$,

(3) $L f_{s, k}=\left(\lambda_{1}+\lambda_{2}+s+k\right) f_{s, k}$.

For fixed $s=0,1,2, \ldots$, the $\left\{f_{s, k}\right\}$ form an orthonormal basis for a subspace of $K_{0} \otimes K_{0}$, transforming according to the irreducible representation $\uparrow_{\tau_{s}} \lambda_{1}+\lambda_{2}+s$.

Lemma 9:

$$
\uparrow_{l_{1}, \lambda_{1}} \otimes \uparrow l_{l_{2}, \lambda_{2}} \cong \sum_{s=0}^{\infty} \oplus \uparrow \tau_{s_{s}, \lambda_{1}+\lambda_{2}+s}
$$

Lemma 10:

$$
\left\langle f_{s, k s} f_{s^{\prime}, k^{\prime}}\right\rangle=\delta_{s s^{\prime}} \delta_{k k^{\prime}} \frac{(q ; q)_{s}(q ; q)_{k}}{(1-q)^{s+k}\left(-\left(\kappa_{1} / \kappa_{2}\right) q^{\lambda_{2}+1} ; q\right)_{s}} .
$$

From (5.5), we have

$$
\left(\exp _{q} t F_{+}\right) f_{s, 0}=\sum_{k=0}^{\infty} \frac{\left(\tilde{l}_{s} t\right)^{k}}{(q ; q)_{k}} f_{s, k}
$$

and, comparing this result with (5.3),

$$
f_{s, k}(z, w)
$$

$$
\begin{aligned}
& =\tilde{l}_{s}^{-k}\left(q^{\lambda_{2} / 2-s / 2} l_{1} z\right)^{k} z^{s} \sum_{r} \frac{\left(q^{-s} ; q\right)_{r}}{\left(-\left(\kappa_{1} / \kappa_{2}\right) q^{\lambda_{2}+1} ; q\right)_{r}(q ; q)_{r}} \\
& \times\left(\frac{l_{1} w q^{\lambda_{1} / 2+\lambda_{2} / 2+s+k}}{z}\right)^{r} \\
& \times_{3} \phi_{2}\left(\begin{array}{cccc}
q^{-r}, & q^{-k}, & -\frac{\kappa_{2}}{\kappa_{1}} q^{-r-\lambda_{2}} & ; q ; \frac{q}{\kappa_{2} l_{2}} \\
q^{1-r-s}, & 0
\end{array}\right.
\end{aligned}
$$

We can use this result to expand the orthonormal basis $\left\{e_{n}^{s}\right\}$ for $K_{0} \otimes K_{0}$,

$$
e_{k}^{s}=\left\|f_{s, k}\right\|^{-1} f_{s, k}, \quad s, k=0,1,2, \ldots,
$$

in terms of the orthonormal basis

$$
\begin{aligned}
& e_{n_{1}} \otimes e_{n_{2}}=\left\|p_{n_{1} n_{2}}\right\|^{-1} p_{n_{1} n_{2}}, \\
& e_{k}^{s}=\sum_{n_{1} n_{2}}\left[\begin{array}{ccc}
l_{1}, \lambda_{1} ; & l_{2} \lambda_{2} ; & s \\
n_{1} ; & n_{2} ; & k
\end{array}\right]_{q} e_{n_{1}} \otimes e_{n_{2}} .
\end{aligned}
$$

These Clebsch-Gordan coefficients vanish unless $n_{1}+n_{2}$ $=s+k$. Furthermore, they satisfy the identities

$\sum_{n_{1}, n_{2}}\left[\begin{array}{ccc}l_{1}, \lambda_{1} ; & l_{2}, \lambda_{2} ; & s \\ n_{1} ; & n_{2} ; & k\end{array}\right]_{q}\left[\begin{array}{ccc}l_{1}, \lambda_{1} ; & l_{2}, \lambda_{2} ; & s^{\prime} \\ n_{1} ; & n_{2} ; & k^{\prime}\end{array}\right]_{q}=\delta_{k k^{\prime}}$,

$\sum_{s, k}\left[\begin{array}{ccc}l_{1}, \lambda_{1} ; & l_{2}, \lambda_{2} ; & s \\ n_{1} ; & n_{2} ; & k\end{array}\right]_{q}\left[\begin{array}{ccc}l_{1}, \lambda_{1} ; & l_{2}, \lambda_{2} ; & s \\ n_{1}^{\prime} ; & n_{2}^{\prime} ; & k\end{array}\right]_{q}=\delta_{n_{1} n_{1}^{\prime},}$ 
where $n_{1}+n_{2}=n_{1}^{\prime}+n_{2}^{\prime}=s+k=s^{\prime}+k^{\prime}$, and we are assuming that $l_{1}, l_{2}>0$ and $\lambda_{1}, \lambda_{2}$ are real. Explicitly, we find

$$
\begin{aligned}
& {\left[\begin{array}{ccc}
l_{1}, \lambda_{1} ; & l_{2}, \lambda_{2} ; & s \\
n_{1} ; & n_{2} ; & k
\end{array}\right]_{q} } \\
&=\tilde{l}_{s}^{-k}\left(q^{\lambda_{2} / 2-s / 2} l_{1}\right)^{k}\left(q^{\lambda_{1} / 2+\lambda_{2} / 2+s+k} l_{1}\right)^{n_{2}}\left(q^{-s} ; q\right)_{n_{2}} \\
& \quad \times\left[\frac{\left(-\left(\kappa_{1} / \kappa_{2}\right) q^{\lambda_{2}+1} ; q\right)_{s}(q ; q)_{n_{1}} \kappa_{2}^{n_{2}}}{\left(-\left(\kappa_{1} / \kappa_{2}\right) q^{\lambda_{2}+1} ; q\right)_{n_{2}}(q ; q)_{n_{2}}(q ; q)_{s}(q ; q)_{k}}\right]^{1 / 2} \\
& \times{ }_{3} \phi_{2}\left(\begin{array}{ccc}
q^{-n_{2}}, & q^{-k}, & -\frac{\kappa_{2}}{\kappa_{1}} q^{-n_{2}-\lambda_{2}} ; q ; \frac{q}{\kappa_{2} l_{2}}
\end{array}\right) .
\end{aligned}
$$

\section{THE QUANTUM ALGEBRA $W_{p}(1)$}

Another $q$ analog of the enveloping algebra of the oscillator algebra is the quantum algebra $W_{p}(1), 2,13,16-21$ generated by the three elements $H^{\prime}, E_{+}^{\prime}, E_{-}^{\prime}$ with the commutation relations

$$
\begin{aligned}
& {\left[H^{\prime}, E_{+}^{\prime}\right]=E_{+}^{\prime}, \quad\left[H^{\prime}, E_{-}^{\prime}\right]=-E_{-}^{\prime},} \\
& {\left[E_{+}^{\prime}, E_{-}^{\prime}\right]=-\frac{p^{1 / 2 H^{\prime}}+p^{-1 / 2 H^{\prime}}}{p^{1 / 4}+p^{-1 / 4}},}
\end{aligned}
$$

where $0<p<1$. The center of this algebra is generated by

$$
\mathscr{C}^{\prime}=p^{1 / 4} p^{1 / 2 H^{\prime}}-p^{3 / 4} p^{-1 / 2 H^{\prime}}+(1-p) E_{+}^{\prime} E_{-}^{\prime} .
$$

$W_{p}(1)$ admits a class of algebraically irreducible representations $\uparrow_{\lambda}^{\prime}$ where $\lambda$ is a complex number. These representations are defined on a space with basis $\left\{e_{n}: n\right.$ $=0,1,2, \ldots\}$, such that

$E_{+}^{\prime} e_{n}$

$$
=p^{-(\lambda+n-1 / 2) / 4}\left[\frac{\left(1-p^{(n+1) / 2}\right)\left(1+p^{\lambda+n / 2}\right)}{1-p}\right]^{1 / 2} e_{n+1},
$$

$E_{-}^{\prime} e_{n}$

$$
=p^{-(\lambda+n-3 / 2) / 4}\left[\frac{\left(1-p^{n / 2}\right)\left(1+p^{\lambda+(n-1) / 2}\right)}{1-p}\right]^{1 / 2} e_{n-1},
$$

$H^{\prime} e_{n}=(\lambda+n) e_{n}$

If $\lambda$ is real then $\dagger_{\lambda}^{\prime}$ is defined on the Hilbert space $K_{0}$ with orthonormal basis $\left\{e_{n}\right\}$, and we have $E_{+}^{\prime}=\left(E_{-}^{\prime}\right)^{*}$, and
$\left(H^{\prime}\right)^{*}=H^{\prime}$. A second convenient basis for $K_{0}$ is $\left\{f_{n}: n\right.$ $=0,1, \ldots\}$, where

$$
\begin{aligned}
& E_{+}^{\prime} f_{n}=p^{(1 / 2-\lambda) / 4}\left(p^{-n / 4}+p^{\lambda+n / 4}\right) f_{n+1}, \\
& E_{-} f_{n}=p^{(3 / 2-\lambda) / 4} \frac{p^{-n / 4}-p^{n / 4}}{1-p} f_{n-1}, \\
& H^{\prime} f_{n}=(\lambda+n) f_{n}, \quad n=0,1, \ldots .
\end{aligned}
$$

In this case,

$$
f_{n}=\left[\frac{\left(p^{1 / 2} ; p^{1 / 2}\right)_{n}}{\left(-p^{\lambda} ; p^{1 / 2}\right)_{n}(1-p)^{n}}\right]^{1 / 2} e_{n}
$$

Corresponding to the irreducible representation $\uparrow_{\lambda}^{\prime}$, we have $\mathscr{C}^{\prime}=p^{(3 / 2-\lambda) / 2}\left(p^{\lambda-1 / 2}-1\right) I$.

In the special case $\lambda=\frac{1}{2}$, expressions (4.2) and (6.2) for the representations $\uparrow_{q}^{1 / 4}, 1 / 2$ and $\uparrow_{1 / 2}^{\prime}$, respectively, of the two $q$ analogs of the oscillator algebra, take on almost the same form when $q=p$. Indeed, we have

$$
\begin{aligned}
& E_{+}^{\prime}=E_{+} q^{(H+1 / 2) / 4}, \quad E_{-}^{\prime}=E_{-} q^{(H-1 / 2) / 4}, \\
& H^{\prime}=H, \quad I=\mathscr{C} .
\end{aligned}
$$

In general, however, these analogs are distinct, the first motivated by the recurrence relations for the functions ${ }_{1} \phi_{1}$ and ${ }_{2} \phi_{1}$, the second by the raising and lowering operators for bosons.

On the other hand, if we consider $\mathrm{U}_{q}\left(\mathrm{su}_{2}\right)$ and $W_{p}(1)$ to be algebras (with identity) over the complex numbers then $\mathrm{U}_{p^{1 / 2}}\left(\mathrm{su}_{2}\right) \equiv W_{p}(1)$. Indeed, if we set

$$
\begin{aligned}
& E_{+}^{\prime}=\frac{e^{i \pi / 4}}{q+1} E_{+}, \quad E_{-}^{\prime}=e^{i \pi / 4}(q-1) E_{-}, \\
& H^{\prime}=H-\frac{i \pi}{2 \ln q}, \quad q=p^{1 / 2},
\end{aligned}
$$

Eqs. (2.1) and (6.1) are identical. Thus the basic facts about tensor products of representations of the form $\uparrow_{\lambda}^{\prime}$ can be obtained easily from the results of Sec. III.

We remark that Biedenharn and Tarlini $^{22}$ have shown how to extend the notion of tensor operators for a Lie algebra to $q$-tensor operators for a quantum algebra in such a way that a generalized Wigner-Eckart theorem holds. This relates to some of our $q$-algebra models.

\section{GENERATING FUNCTIONS, ORTHOGONALITY RELATIONS, AND "ADDITION FORMULAS"}

Now we will present some examples to show how the various models of $q$-algebra representations can be used to derive identities obeyed by a $q$ series associated with the models. We will select all our examples from models 
of "oscillator algebra" representations, as studied in Sec. IV. Examples associated with $\mathrm{U}_{q}\left(\mathrm{su}_{2}\right)$ can be produced by a similar procedure.

We will be concerned with the irreducible representation $\uparrow_{l, \lambda}$ defined by expressions (4.3) with respect to the orthogonal basis $\left\{f_{n}\right\}$ of the Hilbert space $K_{0}$. In analogy with a standard relationship between special functions and the representations of Lie groups, we shall compute the "matrix elements" of $q$ analogs of the group operators $e^{\beta E_{+}} e^{\alpha E_{-}}$, with respect to the $\left\{f_{n}\right\}$ basis. Of course, there are many $q$ analogs of the exponential mapping, none of which have all the properties needed to ensure that there is a true "group" associated with the $q$ algebra. Among the $q$ analogs we shall limit ourselves to the two that are most important: ${ }^{14}$

$e_{q}(A)=\sum_{k=0}^{\infty} \frac{A^{k}}{(q ; q)_{k}}, \quad E_{q}(A)=\sum_{k=0}^{\infty} \frac{q^{k(k-1) / 2}}{(q ; q)_{k}} A^{k}$

If $A$ is a complex number, the first series converges to $1 /(A ; q)_{\infty}$ for $|A|<1$ and the second series converges to $(-A ; q)_{\infty}$ for all $A$. For our first example we consider the matrix elements $T_{n m}(\alpha, \beta)$ of the operator $E_{q}\left(\beta E_{+}\right) e_{q}\left(\alpha E_{-}\right)$:

$$
E_{q}\left(\beta E_{+}\right) e_{q}\left(\alpha E_{-}\right) f_{m}=\sum_{n=0}^{\infty} T_{n m}(\alpha, \beta) f_{n}
$$

It is most convenient to evaluate (7.2) in the model (4.16) in which $f_{n}(z)=q^{n(n+1) / 4} z^{n}$. In this case (7.2) becomes the generating function,

$$
\begin{gathered}
q^{-m(m+1) / 4}\left(\frac{\alpha l}{1-q}\right)^{m}(-\beta l z ; q)_{\infty}\left(\frac{q(1-q) z}{\alpha l} ; q\right)_{m} \\
=\sum_{n=0}^{\infty} T_{n m}(\alpha, \beta) q^{n(n+1) / 4} z^{n},
\end{gathered}
$$

convergent for all $\alpha, \beta$. Thus

$$
\begin{aligned}
T_{n m}(\alpha, \beta)= & \frac{q^{(n-m)(n-m-1) / 4}(q ; q)_{m}\left(\beta l q^{-m / 2-1 / 2}\right)^{n-m}}{(q ; q)_{n}} \\
& \times L_{m}^{(n-m)}\left(\frac{-\alpha \beta l^{2} q^{-1-m}}{1-q} ; q\right) \\
= & q^{-m(m+1) / 4+n(n+1) / 4}\left(\frac{\alpha l}{1-q}\right)^{m-n} \\
& \times L_{n}^{(m-n)}\left(\frac{-\alpha \beta l^{2} q^{-1-m}}{1-q} ; q\right),
\end{aligned}
$$

where $e^{14,23,24}$

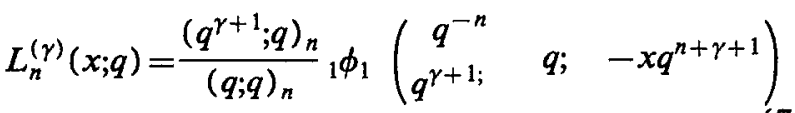

is a $q$-Laguerre polynomial. [Note that $L_{m}^{(n-m)}(x ; q)$ $=(-x)^{(m-n)}\left((q ; q)_{n} /(q ; q)_{m}\right) L_{n}^{(m-n)}(x ; q)$.]

We can obtain the matrix elements of the operator $e_{q}\left(\beta E_{+}\right) E_{q}\left(\alpha E_{-}\right)$for free, since $\left(E_{q}\left(\beta E_{+}\right) e_{q}\left(\alpha E_{-}\right)\right)^{*}$ $=e_{q}\left(\bar{\alpha} E_{+}\right) E_{q}\left(\bar{\beta} E_{-}\right)$. Defining matrix elements $S_{n m}(\alpha, \beta)$ by

$$
e_{q}\left(\beta E_{+}\right) E_{q}\left(\alpha E_{-}\right) f_{m}=\sum_{n=0}^{\infty} S_{n m}(\alpha, \beta) f_{n},
$$

we find

$$
S_{n m}(\alpha, \beta)=\frac{(q ; q)_{m}}{(q ; q)_{n}}(1-q)^{n-m} \overline{T_{n m}(\bar{\alpha}, \bar{\beta})},
$$

so, if $l$ is real,

$$
\begin{aligned}
S_{n m}(\alpha, \beta)= & q^{n(n+1) / 4-m(m+1) / 4} \frac{(q ; q)_{n}}{(q ; q)_{m}}(\beta l)^{m-n} \\
& \times L_{n}^{(m-n)}\left(-\frac{\alpha \beta l^{2} q^{-1-m}}{1-q} ; q\right) .
\end{aligned}
$$

From the explicit expression (7.5), we can verify the recurrence relations

$$
\begin{gathered}
\frac{1}{x}\left(1-T_{x}^{-1}\right) L_{k}^{(\gamma)}(x ; q)=q^{\gamma} L_{k-1}^{(\gamma+1)}(x ; q), \\
\left(1-q^{\gamma}(1+x) T_{x}\right) L_{k}^{(\gamma)}(x ; q)=\left(1-q^{k+1}\right) L_{k+1}^{(\gamma-1)}(x ; q), \\
k=0,1, \ldots,
\end{gathered}
$$

where we adopt the convention that $L_{-1}^{(\gamma)}(x ; q) \equiv 0$. Thus the operators

$$
\begin{aligned}
& E_{+}^{\prime}=l t\left(1-(1+x) T_{t}^{-1} T_{x}\right), \quad H^{\prime}=t \partial_{t}, \\
& E_{-}^{\prime}=\frac{l q^{\lambda}}{(1-q) x t}\left(1-T_{x}^{-1}\right), \quad \mathscr{E}^{\prime}=l^{2} q^{\lambda-1},
\end{aligned}
$$

and the basis functions

$$
\begin{gathered}
f_{n}(x, t)=(q ; q)_{n} q^{n(n+1) / 4} L_{n}^{(-\lambda-n)}(x ; q) t^{\lambda+n}, \\
n=0,1, \ldots,
\end{gathered}
$$

define a two-variable model of $\uparrow_{l, \lambda}$, i.e., they satisfy relations (4.3). For fixed $m$, we see from (7.8) that the matrix elements $S_{m n}(\alpha, \beta)$ are the special case of this model, where $\lambda=-m$, and we have the identifications 


$$
t=\frac{1}{\beta l}, \quad x=-\frac{\alpha \beta l^{2}}{(1-q) q^{1+m}} .
$$

Using Lemma 4, we find that the action of the operators $E_{q}\left(\beta E_{+}^{\prime}\right) e_{q}\left(\alpha E_{-}^{\prime \prime}\right.$ on the monomial $x^{m} t^{\delta}(x t \neq 0)$ is

$$
\begin{aligned}
& E_{q}\left(\beta E_{+}^{\prime}\right) e_{q}\left(\alpha E_{-}^{\prime}\right) x^{m} t^{\delta} \\
& =x^{m} t^{\delta} \frac{\left(l \alpha q^{\lambda-m} / t x ; q\right)_{\infty}(-l \beta t ; q)_{\infty}\left(l \beta t x q^{-\delta+m} ; q\right)_{\infty}}{\left(l \alpha q^{\lambda} / t x ; q\right)_{\infty}\left(-l \beta t q^{-\delta+m} ; q\right)_{\infty}},
\end{aligned}
$$

for $|\alpha|,|\beta|$ sufficiently small. From this result, (7.2) and (7.11), we obtain the generating function

$$
\begin{aligned}
& E_{q}\left(\beta E_{+}^{\prime}\right) e_{q}\left(\alpha E_{-}^{\prime}\right) f_{m}(x, t) \\
& =\frac{q^{m(m+1) / 4}\left(q^{-\lambda+1} ; q\right)_{n}(-l \beta t ; q)_{\infty}\left(l \beta t x q^{-\lambda-m_{m}} ; q\right)_{\infty}}{\left(-l \beta t q^{-\lambda-m_{;}} ; q\right)_{\infty}} \\
& \quad \times t^{\lambda+m} \\
& { }_{3} \phi_{2}\left(\begin{array}{l}
q^{-m}, \quad \frac{t x q^{1-\lambda}}{l \alpha},-l \beta t q^{-\lambda-m} ; q ; \\
q^{-\lambda+1}, l \beta t x q^{-\lambda-m}
\end{array}\right) \\
& =\sum_{n=0}^{\infty} T_{n m}(\alpha, \beta) f_{n}(x, t), \\
& \left|\frac{l \alpha q^{\lambda}}{t x}\right|<1, \quad\left|l \beta t q^{-\lambda-m}\right|<1 .
\end{aligned}
$$

In the special case where the $\left\{f_{n}\right\}$ basis reduces to the $\left\{S_{n m}\right\}$ basis, we can view (7.14) as a $q$ analog of an addition theorem for Laguerre polynomials. ${ }^{1}$

Relations (7.9) can be used to derive orthogonality relations for the $q$-Laguerre polynomials. Let $S^{\gamma}$ be the space of all real polynomials in $x$ with inner product

$$
\langle\Psi, \Theta\rangle_{\gamma}=\int_{0}^{\infty} \Psi(x) \Theta(x) \rho_{\gamma}(x) d x, \quad \Psi, \Theta \in S^{\gamma},
$$

where $\rho_{\gamma}(x)$ is a weight function to be determined. From (7.9) we define operators

$$
R_{\gamma}: S^{\gamma} \rightarrow S^{\gamma+1}, \quad L_{\gamma}: S^{\gamma} \rightarrow S^{\gamma-1},
$$

by

$$
R_{\gamma}=(1 / x)\left(1-T_{x}^{-1}\right), \quad L_{\gamma}=1-q^{\gamma}(1+x) T_{x}
$$

Furthermore, we require that

$$
\left\langle R_{\gamma} \Psi, \Theta\right\rangle_{\gamma+1}=\left\langle\Psi, L_{\gamma+1} \Theta\right\rangle_{\gamma}
$$

for all $\Psi \in S^{\gamma}, \Theta \in S^{\gamma+1}$. This leads to the conditions

$$
\rho_{\gamma+1}(x)=x \rho_{\gamma}(x), \quad \rho_{\gamma}(q x)=q^{\gamma}(1+x) \rho_{\gamma}(x) .
$$

The solution, unique up to multiplication by a function of $\gamma$ alone, is

$$
\rho_{\gamma}(x)=x^{\gamma} /(-x ; q)_{\infty},
$$

where we require $\gamma>-1$ for convergence of the inner product. It follows that the operators $T_{\gamma}$ $=R_{\gamma-1} L_{\gamma}: S^{\gamma} \rightarrow S^{\gamma}$ are self-adjoint and map polynomials of order $m$ to polynomials of the same order. From (7.9), we see that the polynomials $L_{k}^{(\gamma)}(x ; q), k=0,1, \ldots$, are exactly the eigenfunctions of $T_{\gamma}$, and that they correspond to the eigenvalues $q^{\gamma}\left(1-q^{k}\right)$. Since eigenfunctions of $T_{\gamma}$ corresponding to distinct eigenvalues must be orthogonal, we have

$$
\left\langle L_{n}^{(\gamma)}, L_{m}^{(\gamma)}\right\rangle_{\gamma}=\delta_{m n} A_{n}^{\gamma}
$$

We can also use the recurrence relations (7.9) to help determine $A_{n}^{\gamma}$. Setting $\Psi=L_{k+1}^{(\gamma)}, \Theta=L_{k}^{(\gamma+1)}$ in (7.16), we find $q^{\gamma}\left\|L_{k}^{(\gamma+1)}\right\|_{\gamma+1}^{2}=\left(1-q^{k+1}\right)\left\|L_{k+1}^{(\gamma)}\right\|_{\gamma}^{2}$. Hence

$$
\left\|L_{k}^{(\gamma)}\right\|_{\gamma}^{2}=\frac{q^{\gamma k+k(k-1) / 2}}{(q ; q)_{k}}\|1\|_{\gamma+k}^{2} .
$$

Furthermore, from the explicit expressions for $L_{k}^{(\gamma)}$ and $\rho_{\gamma}$ we can write the identity $\left\langle L_{1}^{(\gamma)}, L_{0}^{(\gamma)}\right\rangle_{\gamma}=0$ in the form $\|1\|_{\gamma+1}^{2}=-\left(1-q^{-\gamma-1}\right)\|1\|_{\gamma}^{2}$ It follows that $\|1\|_{\gamma+k}^{2}=\left(q^{\gamma+1} ; q\right)_{k} q^{-\gamma k} q^{-k(k+1) / 2}\|1\|_{\gamma}^{2}$, so

$$
A_{n}^{\gamma}=\frac{\left(q^{\gamma+1} ; q\right)_{n}}{(q ; q)_{n} q^{n}}\|1\|_{\gamma}^{2}
$$

A straightforward contour integration argument gives

$$
\begin{gathered}
\|1\|_{\gamma}^{2}=-\frac{\pi\left(q^{-\gamma} ; q\right)_{\infty}}{\sin \pi \gamma(q ; q)_{\infty}}, \quad\|1\|_{k}^{2}=\ln q^{-1}(q ; q)_{k} q^{-k(k+1) / 2}, \\
k=0,1,2, \ldots
\end{gathered}
$$

(For related works on orthogonality extending that of the classical polynomials see Refs. 25-31.)

There are also $q$ analogs of the orthogonality relations for matrix elements of the oscillator group. In expressions (7.4) and (7.8) for the matrix elements we can restrict the parameters $\alpha, \beta$ so that $\alpha=r e^{i \theta}, \beta=r e^{-i \theta}$, where $r \geqslant 0$ and $\theta$ is real. Setting $S_{m n}(\alpha, \beta) \equiv S_{m n}[r, \theta]$, $T_{m n}(\alpha, \beta) \equiv T_{m n}[r, 0]$, it is easy to see that

$$
\left(S_{m n}, S_{m^{\prime} n^{\prime}}\right)=\left(T_{m n}, T_{m^{\prime} n^{\prime}}\right)=0,
$$

unless $m=m^{\prime}, n=n^{\prime}$, where $(\cdot, \cdot)$ is the inner product,

$$
(f, g)=\int_{0}^{2 \pi} d \theta \int_{0}^{\infty} \frac{r^{2 \alpha} d r^{2}}{\left(-r^{2} ; q\right)_{\infty}} f[r, \theta] \overline{g[r, \theta]} .
$$


We note that there exist many inner products, in addition to (7.18), for which the Laguerre polynomials $\left\{L_{k}^{(\alpha)}: k=0,1, \ldots\right\}$ are orthogonal. Indeed one can use a technique analogous to the derivation of (3.10) to obtain a family of orthogonality relations with discrete weight functions Refs. 23, 24, and 14, p. 194).

As a second example of the computation of matrix elements we consider the operator $e_{q}\left(\beta E_{+}\right) e_{q}\left(\alpha E_{-}\right)$:

$$
e_{q}\left(\beta E_{+}\right) e_{q}\left(\alpha E_{-}\right) f_{m}=\sum_{n=0}^{\infty} A_{n m}(\alpha, \beta) f_{n}
$$

The result is

$$
\begin{aligned}
& A_{n m}(\alpha, \beta)=\frac{q^{m(m-1) / 4-n(n-1) / 4}(\beta l)^{n-m}}{(q ; q)_{n-m}}
\end{aligned}
$$

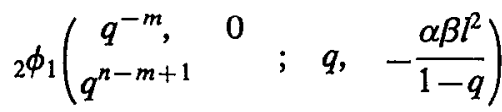

$$
\begin{aligned}
& =\frac{q^{n(n+3) / 4-m(m+3) / 4}(q ; q)_{m}}{(q ; q)_{m-n}(q ; q)_{n}}\left(\frac{\alpha l}{1-q}\right)^{m-n}
\end{aligned}
$$

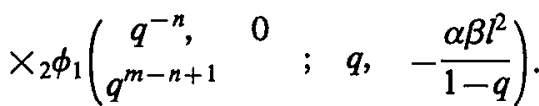

These functions are essentially the Wall polynomials. ${ }^{3}$

From the power series representation of the ${ }_{2} \phi_{1}$ polynomials, we can verify the recurrence relations

$$
\begin{aligned}
& (1 / x)\left(T_{x}-1\right) \widetilde{L}_{k}^{(\gamma)}(x ; q)=q^{-k} \widetilde{L}_{k-1}^{(\gamma+1)}(x ; q), \\
& \left(-q^{\gamma}+T_{x}^{-1}-q^{-1} x T_{x}^{-1}\right) \widetilde{L}_{k}^{(\gamma)}(x ; q) \\
& =\left(1-q^{k+1}\right) \widetilde{L}_{k+1}^{(\gamma-1)}(x ; q),
\end{aligned}
$$

where

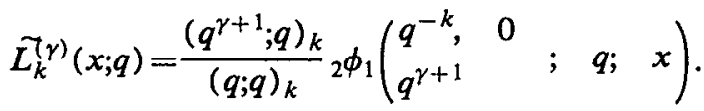

Thus the operators

$$
\begin{aligned}
& \tilde{E}_{+}=\frac{t l}{q^{1 / 2}}\left(-q^{\gamma} T_{t}^{-1}+T_{x}^{-1}-q^{-1} x T_{x}^{-1}\right), \quad \tilde{H}=t \partial_{t}+\lambda \\
& \tilde{E}_{-}=\frac{q^{1 / 2} l}{(1-q) t x}\left(T_{x}-1\right), \quad \widetilde{E}=l^{2} q^{\lambda-1},
\end{aligned}
$$

and the basis functions

$$
f_{n}(x, t)=(q ; q)_{n} q^{n(n-1) / 4} \widetilde{L}_{n}^{(\gamma-n)}(x ; q) t^{n}
$$

define a two-variable model of $\uparrow_{l, \lambda}$ for each fixed complex number $\gamma$. Note that the matrix elements $A_{n m}(\alpha, \beta)$ are the special case of this model, for which $\gamma=m$ and

$$
t=\frac{1}{\beta l}, \quad x=-\frac{\alpha \beta l^{2}}{(1-q)} .
$$

We can use relations (7.24) to derive orthogonality relations for the $q$ analogs $\widetilde{L}_{k}^{(\gamma)}(x ; q)$ of the Laguerre polynomials. Let $S^{\gamma}$ be the space of all real polynomials in $x$ with discrete inner product

$$
\langle\Psi, \Theta\rangle_{\gamma}=\int_{0}^{c} \Psi(x) \Theta(x) \rho_{\gamma}(x) d_{q} x
$$

[see (3.9)], where $c$ and $\rho_{\gamma}(x)$ are to be determined. From (7.24), we define operators

$$
R_{\gamma}: S^{\gamma} \rightarrow S^{\gamma+1}, \quad L_{\gamma}: S^{\gamma} \rightarrow S^{\gamma-1},
$$

by

$$
R_{\gamma}=(1 / x)\left(T_{x}-1\right), \quad L_{\gamma}=-q^{\gamma}+T_{x}^{-1}-q^{-1} x T_{x}^{-1} .
$$

We require that relations $(7.16)$ hold:

$$
\left\langle R_{\gamma} \Psi, \Theta\right\rangle_{\gamma+1}=\left\langle\Psi, L_{\gamma+1} \Theta\right\rangle_{\gamma},
$$

for all $\Psi \in S^{\gamma}, \Theta \in S^{\gamma+1}$. This leads to the conditions

$c=q, \quad \rho_{\gamma+1}(x)=q^{\gamma+1} x \rho_{\gamma}(x), \quad \rho_{\gamma}(q x)=\frac{q^{\gamma}}{1-x} \rho_{\gamma}(x)$.

We choose the solution

$$
\rho_{\gamma}(x)=(x ; q)_{\infty} x^{\gamma} q^{\gamma(\gamma+1) / 2},
$$

where we require $\gamma>-1$ for convergence of the inner product. The operators $T_{\gamma}=R_{\gamma-1} L_{\gamma}: S^{\gamma} \rightarrow S^{\gamma}$ are selfadjoint with eigenfunctions $\widetilde{L}_{k}^{(\gamma)}(x ; q)$ and eigenvalues $q^{\gamma}\left(1-q^{k}\right), k=0,1, \ldots$. Hence we must have

$$
\left\langle\tilde{L}_{n}^{(\gamma)}, \tilde{L}_{m}^{(\gamma)}\right\rangle_{\gamma}=\delta_{m n} B_{n}^{\gamma}
$$

Exactly as in the proof of (7.19), the recurrence relations (7.25) yield the formula

$$
\left\|\widetilde{L}_{k}^{(\gamma)}\right\|_{\gamma}^{2}=\frac{q^{-k(k-1) / 2}}{(q ; q)_{k}}\|1\|_{\gamma+k}^{2}
$$

From the explicit expressions for $\widetilde{L}_{k}^{(\gamma)}$ and $\rho_{\gamma}$ we can write the identity $\left\langle\widetilde{L}_{1}^{(\gamma)}, \widetilde{L}_{0}^{(\gamma)}\right\rangle_{\gamma}=0$ in the form $\|1\|_{\gamma+1}^{2}=q^{\gamma+2}(1$ $\left.-q^{\gamma+1}\right)\|1\|_{\gamma}^{2}$ Thus $\|1\|_{\gamma+k}^{2}=\left(q^{\gamma+1} ; q\right)_{k} q^{\gamma k+k(k+3) / 2}\|1\|_{\gamma}^{2}$ and 


$$
B_{n}^{\gamma}=\frac{\left(q^{\gamma+1} ; q\right)_{n} q^{(\gamma+2) n}}{(q ; q)_{n}}\|1\|_{\gamma}^{2}
$$

It is easy to evaluate the sum $\|1\|_{\gamma}^{2}$ directly:

$$
\|1\|_{\gamma}^{2}=\int_{0}^{q} \rho_{\gamma}(x) d_{q} x=\frac{q(1-q)(q ; q)_{\infty}}{\left(q^{\gamma+1} ; q\right)_{\infty}} q^{\gamma(\gamma+3) / 2} .
$$

In a manner similar to the derivation of (7.21) we can also obtain orthogonality relations for the matrix elements $A_{m n}$. [We note that recurrence relations of the type (7.9), (7.24) are closely related to the factorization method. ${ }^{25,32}$ ]

'W. Miller, Lie Theory and Special Functions (Academic, New York, 1986).

${ }^{2}$ L. Floreanini and L. Vinet, " $q$-Orthogonal polynomials and the oscillator quantum group," INFN preprint, Trieste, AE-90/23 (1990).

${ }^{3} \mathrm{~T}$. H. Koornwinder, "The addition formula for little $q$-Legendre polynomials and the SU(2) quantum group," SIAM J. Math. Anal. 22, 295 (1991).

${ }^{4}$ T. H. Koornwinder, "Representations of the twisted SU(2) quantum group and some $q$-hypergeometric orthogonal polynomials," Ned. Akad. Wetensch. Proc. Ser. A 92, 97 (1989).

${ }^{5}$ T. Masuda, K. Mimachi, Y. Nakagami, M. Noumi, Y. Saburi, and K. Ueno, "Unitary representations of the quantum groups $\mathrm{SU}_{q}(1,1)$ : Structure of the dual space of $\mathscr{U}_{q}(s i(2)), "$ Lett. Math. Phys. 19, 187 (1990).

${ }^{6}$ T. Masuda, K. Mimachi, Y. Nakagami, M. Noumi, Y. Saburi, and K. Ueno, "Unitary representations of the quantum groups $S U_{q}(1,1)$ : II. Matrix elements of unitary representations and the basic hypergeometric functions," Lett. Math. Phys. 19, 194 (1990).

"M. Jimbo, "A $q$-difference analogue of $U(g)$ and the Yang-Baxter equation," Lett. Math. Phys. 10, 63 (1985).

${ }^{8}$ I. I. Kachurik and A. U. Klimyk, "On Clebsch-Gordan coefficients of quantum algebra $\mathrm{U}_{q}\left(\mathrm{SU}_{2}\right)$," preprint (1989).

${ }^{9}$ I. I. Kachurik and A. U. Klimyk, "Asymptotic properties of ClebschGordan and Racah coefficients and Racah coefficients of the quantum algebra $\mathrm{U}_{4}\left(\mathrm{su}_{2}\right)$, preprint (1990).

${ }^{10} \mathrm{~S}$. L. Woronowicz, "Twisted SU(2) group. An example of a noncommutative differential calculus," Publ. RIMS, Kyoto Univ. 23, 117 (1987).

"B. Jurco, "On coherent states for the simplest quantum groups," Lett. Math. Phys. 21, 51 (1991).
${ }^{12}$ H. T. Koelink and T. H. Koornwinder, "The Clebsch-Gordan coefficients for the quantum group $S_{\mu} U(2)$ and $q$-Hahn polynomials, CWI Report, 1989.

${ }^{13}$ P. P. Kulish and E. V. Damashinsky, "On the $q$ oscillator and the quantum algebra $\mathrm{su}_{q}(1,1)$," J. Phys. A 23, L415 (1990).

${ }^{14} \mathrm{G}$. Gasper and M. Rahman, Basic Hypergeometric Series (Cambridge U.P., Cambridge, 1990).

${ }^{15}$ E. Abe, Hopf Algebras (Cambridge U.P., Cambridge, 1980).

${ }^{16} \mathrm{~L}$. C. Biedenharn, "The quantum group $S U(2)_{q}$ and a $q$-analog of the boson operators," J. Phys. A 22, L873 (1989).

${ }^{17} \mathrm{~L}$. Floreanini and L. Vinet, " $q$-analogues of the parabose and parafermi oscillators and representations of quantum algebras," J. Phys. A 23, L1019 (1990).

${ }^{18} \mathrm{~T}$. Hayashi, " $q$-analogues of Clifford and Weyl algebras. Spinor and oscillation representations of quantum enveloping algebras," Commun. Math. Phys. 127, 129 (1990).

${ }^{19}$ A. J. Macfarlane, "On $q$-analogues of the quantum harmonic oscillator and the quantum group $S U(2)$," J. Phys. A 22, 4581 (1989).

${ }^{20} \mathrm{H}$. Ui and N. Aizawa, "The $q$-analogue of boson commutator and the quantum groups $S U_{q}(2)$ and $S U_{q}(1,1)$," Mod. Phys. Lett. A 5, 237 (1990).

${ }^{21} \mathrm{H}$. Yan, " $q$-deformed oscillator algebra as a quantum group," Academia Sinica preprint, AS-ITP-90-33 (1990).

${ }^{22} \mathrm{~L}$. C. Biedenham and M. Tarlini, "On $q$-tensor operators for quantum groups," Lett. in Math. Phys. 20, 271 (1990).

${ }^{23}$ G. E. Andrews and R. Askey, Classical Orthogonal Polynomials, Lecture Notes No. 1171 (Springer-Verlag, New York, 1985), pp. 36-62.

${ }^{24}$ D. S. Moak, "The $q$-analogue of the Laguerre polynomials," J. Math. Anal. Appl. 81, 20 (1981).

${ }^{25}$ A. K. Agarwal, E. G. Kalnins, and W. Miller, "Canonical equations and symmetry techniques for $q$-series," SIAM J. Math. Anal. 18, 1519 (1987).

${ }^{26} \mathrm{R}$. Askey and J. Wilson, "Some basic hypergeometric orthogonal polynomials that generalize Jacobi polynomials," Memoirs of the AMS, No. 319, 1985.

${ }^{27} \mathrm{~W}$. Hahn, "Uber Orthogonal polynome, die $q$-Differenzengleichen genugen," Math. Nach. 2, 4 (1949).

${ }^{28} \mathrm{E}$. G. Kalnins and W. Miller, "Symmetry techniques for $q$-series: Askey-Wilson polynomials," Rocky Mtn. J. Math. 19, 223 (1989).

${ }^{29}$ W. Miller, "A note on Wilson polynomials," SIAM J. Math. Anal. 18, 1221 (1987).

${ }^{30}$ A. F. Nikiforov and S. K. Suslov, "Classical orthogonal polynomials of a discrete variable on nonuniform lattices," Lett. Math. Phys. 11, 27 (1986).

${ }^{31}$ A. F. Nikiforov, S. K. Suslov, and V. B. Uvarov, Classical Orthogonal Polynomials of a Discrete Variable (Nauka, Moscow, 1985) (in Russian).

${ }^{32} \mathrm{~W}$. Miller, "Lie theory and $q$-difference equations," SIAM J. Math. Anal. 1, 171 (1970). 